\title{
PROPER ORTHOGONAL DECOMPOSITION FOR OPTIMALITY SYSTEMS
}

\author{
KARL KUnisch $^{1}$ And Stefan VOLKWEIN ${ }^{1}$
}

\begin{abstract}
Proper orthogonal decomposition (POD) is a powerful technique for model reduction of non-linear systems. It is based on a Galerkin type discretization with basis elements created from the dynamical system itself. In the context of optimal control this approach may suffer from the fact that the basis elements are computed from a reference trajectory containing features which are quite different from those of the optimally controlled trajectory. A method is proposed which avoids this problem of unmodelled dynamics in the proper orthogonal decomposition approach to optimal control. It is referred to as optimality system proper orthogonal decomposition (OS-POD).
\end{abstract}

Mathematics Subject Classification. 35K20, 65Nxx, 90C20.

Received March 24, 2006.

\section{INTRODUCTION}

In this research we focus on a model reduction technique for open loop optimal control problems of the form

$$
\min J(y, u) \quad \text { s.t. } \quad\left\{\begin{array}{l}
y_{t}(t)=F(y(t))+B(u(t)) \text { for } t \in(0, T] \\
y(0)=y_{0} .
\end{array}\right.
$$

Here $J$ denotes a cost-functional depending on the state $y$ and the control $u$, with $y$ and $u$ depending on time $t \in[0, T]$ and the spatial variable $x$ from the domain $\Omega \subset \mathbb{R}^{d}$ with $d \in \mathbb{N}$. The differential equation constraint in $(\mathbf{P})$ will be considered in weak form in a separable Hilbert space $V$ of functions defined on $\Omega$. The precise conditions on the possibly nonlinear operator $F: V \rightarrow V^{*}$ and the control operator $B: U \rightarrow V^{*}$ will be given in the following section. Here $U$ denotes the control space. We utilize a Gelfand triple $V \subset H \subset V^{*}$, with $V$ compactly and densely embedded in the real Hilbert space $H$, and $V^{*}$ denoting the dual space of $V$. Further $y_{0}$ stands for the given initial condition.

While significant advances have been made over the last decade in efficient solving of $(\mathbf{P})$ good reasons remain for applying model reduction techniques to the partial differential equation and solving the optimal control problem for the reduced system. Such a procedure can become necessary in the case a large systems of partial differential equations or in the context of real time requirements, for example. It provides a possibility for obtaining closed loop solutions based on solving the Hamilton-Jacobi-Bellman equation which is otherwise computationally infeasible even for very coarse spatial discretizations.

\footnotetext{
Keywords and phrases. Optimal control, partial differential equations, proper orthogonal decomposition, model reduction.

1 Karl-Franzens-Universität Graz, Institut für Mathematik und Wissenschaftliches Rechnen, Heinrichstrasse 36, 8010 Graz, Austria. karl.kunisch@uni-graz.at; stefan.volkwein@uni-graz.at
} 
To obtain low dimensional models of complex high dimensional systems many different approaches, including balanced truncation and reduced basis methods, were proposed. Here we focus on model reduction based on proper orthogonal decomposition (POD). It is based on a Galerkin technique where the basis functions $\left\{\psi_{i}\right\}_{i=1}^{\ell}$ are chosen as the solutions to

$$
\left\{\begin{array}{l}
\min _{\left\{\psi_{i}\right\}_{i=1}^{\ell}} \int_{0}^{T}\left\|y(t, \cdot)-\sum_{i=1}^{\ell}\left\langle y(t, \cdot), \psi_{i}\right\rangle_{X} \psi_{i}\right\|_{X}^{2} \mathrm{~d} t \\
\text { subject to }\left\langle\psi_{i}, \psi_{j}\right\rangle_{X}=\delta_{i, j} \quad \text { for } 1 \leq i, j \leq \ell
\end{array}\right.
$$

with $X$ a Hilbert space satisfying $V \subset X \subset H$. The basis defined by (1.1) is given by the eigenfunctions corresponding to the $\ell$ largest eigenvalues $\lambda_{i}$ of the following eigenvalue problem:

$$
\begin{aligned}
\mathcal{R} \psi_{i}:=\int_{0}^{T}\left\langle y(t), \psi_{i}\right\rangle_{X} y(t) \mathrm{d} t & =\lambda_{i} \psi_{i} & & \text { for } 1 \leq i \leq \ell, \\
\left\langle\psi_{j}, \psi_{i}\right\rangle_{X} & =\delta_{i j} & & \text { for } 1 \leq i, j \leq \ell,
\end{aligned}
$$

see [9], for example. Let us denote $X^{\ell}=\operatorname{span}\left\{\psi_{i}\right\}_{i=1}^{\ell}$ endowed with the norm induced by $X$. In (1.2) the function $y$ denotes a solution to the dynamical system in $(\mathbf{P})$ computed at a reference control $u$. Hence the Galerkin subspace $X^{\ell}$ depends on the control as well. Typical choices for $X$ are $X=H$ and $X=V$. Our assumptions will guarantee that $X^{\ell} \subset V$. Let $P^{\ell}: H \rightarrow X^{\ell}$ denote the orthogonal projection with respect to the $H$ norm. Since $X^{\ell} \subset V$ the projection $P^{\ell}$ can be extended to a bounded linear mapping from $V^{*} \rightarrow X^{\ell} \subset V^{*}$ satisfying $\left\langle P^{\ell} f-f, \phi\right\rangle_{V^{*}, V}=0$ for all $\phi \in X^{\ell}$ and $f \in V^{*}$. In the POD-approach to (P) the dynamical system is replaced by a Galerkin projection on the POD-subspace $X^{\ell}$. This results in

$$
\min J\left(y^{\ell}, u\right) \quad \text { s.t. } \quad\left\{\begin{array}{l}
y_{t}^{\ell}=P^{\ell}\left(F\left(y^{\ell}(t)\right)+B(u(t))\right) \\
y^{\ell}(0)=P^{\ell} y_{0} .
\end{array} \text { for } t \in(0, T]\right.
$$

The discretization of the control variable $u \in L^{2}(0, T ; U)$ is a different issue that is not the focus of the present research. In Section 2 the control space $U$ will be chosen to be finite dimensional. Note that the projection in $\left(\mathbf{P}^{\ell}\right)$ depends on the state $y$ and hence on the reference control $u$ at which the eigenvalue problem (1.2) is solved for the basis $\left\{\psi_{i}\right\}_{i=1}^{\ell}$. This may deter from one of the main advantages of the POD-approach for model reduction, which consists in the fact that unlike typical finite element basis functions the elements of the POD-basis reflect the dynamics of the system. In optimal control this feature gets lost if the dynamics of the state corresponding to the reference control is significantly different from that of the trajectory corresponding to the optimal control of $(\mathbf{P})$ or the POD-Galerkin approximation $\left(\mathbf{P}^{\ell}\right)$. To eliminate this weakness of the conventional approach we propose to consider

$$
\left\{\begin{array}{l}
\min J\left(y^{\ell}, u\right) \text { subject to } \\
y_{t}^{\ell}(t)=P^{\ell}(u) F\left(y^{\ell}(t)\right)+P^{\ell}(u) B(u(t)), \text { for } t \in(0, T] \\
y^{\ell}(0)=P^{\ell}(u) y_{0} \\
y_{t}(t)=F(y(t))+B(u(t)), \text { for } t \in(0, T] \\
y(0)=y_{0}, \\
\mathcal{R}(y(u)) \psi_{i}=\lambda_{i} \psi_{i} \text { for } 1 \leq i \leq \ell \\
\left\langle\psi_{j}, \psi_{i}\right\rangle_{X}=\delta_{i j} \text { for } 1 \leq i, j \leq \ell
\end{array}\right.
$$


where we now indicate the dependence of the projection $\left(\mathbf{P}^{\ell}\right)$ and the correlation operator $\mathcal{R}$ on the control $u$. The first three lines in $\left(\mathbf{P}_{\text {OS-POD }}^{\ell}\right)$ coincide with $\left(\mathbf{P}^{\ell}\right)$, the next two are the infinite dynamical system and the last two represent the eigenvalue problem characterizing the POD basis. For the optimal solution $\left(y^{\ell *}, y^{*}, \psi_{i}^{*}, \lambda_{i}^{*}, u^{*}\right)$ the problem formulation $\left(\mathbf{P}_{\mathrm{OS}-\mathrm{POD}}^{\ell}\right)$ has the property that the associated POD-reduced system is computed from the trajectory corresponding to the optimal control $u^{*}$ and thus, differently from $\left(\mathbf{P}^{\ell}\right)$, the problem of unmodelled is removed. ( $\left.\mathbf{P}_{\mathrm{OS}-\mathrm{POD}}^{\ell}\right)$ can be considered as an optimization problem in the variables $\left(y^{\ell}, y, \psi_{i}, \lambda_{i}, u\right)$ or, alternatively it can be looked upon in the reduced sense with $u$ the only independent variable, and $y^{\ell}, y, \psi_{i}, \lambda_{i}$ dependent variables defined by the equations in $\left(\mathbf{P}_{\text {OS-POD }}^{\ell}\right)$. In either case $\left(\mathbf{P}_{\text {OS-POD }}^{\ell}\right)$ is more complicated than the original problem and we thus need to justify its scope. We shall show that the optimality system for $\left(\mathbf{P}_{\text {OS-POD }}^{\ell}\right)$ involves two adjoint equations. The adjoint equation for the finite dimensional system is the common one for the Galerkin approach. It contains the linearization of the cost $J$ with respect to the statevariable $y^{\ell}$ as forcing function. The second adjoint equation results from the infinite dimensional system and contains as forcing term information of the linearization of the correlation operator $\mathcal{R}$. The gradient of the reduced functional $u \mapsto J\left(y^{\ell}(u), u\right)$ with $y^{\ell}$ the solution to the POD system in $\left(\mathbf{P}_{\text {OS-POD }}^{\ell}\right)$ can be expressed in terms of the solutions to these two adjoints. This can be utilized for practical realizations of $\left(\mathbf{P}_{\text {OS-POD }}^{\ell}\right)$. It suggests a splitting of the variables $\mathrm{z}$ into $\mathrm{z}_{1}=\left(y^{\ell}, u\right)$ and $\mathrm{z}_{2}=\left(y, \psi_{i}, \lambda_{i}\right)$. Minimizing $J\left(y^{\ell}, u\right)$ with respect to the former for fixed $z_{2}$ results in the common POD-optimization problem for which first order, gradient based, or second order methods can be used. Minimization with respect to the second set of variables requires one additional forward and one adjoint sweep of the full system if gradient iterations are used. Thus for practical realization of $\left(\mathbf{P}_{\text {OS-POD }}^{\ell}\right)$ we suggest a splitting scheme where minimization with respect to the $z_{1}$ is done more accurately than with respect to $z_{2}$. The benefit for this extra work is that the POD basis is updated in the direction of the minimum of $J$.

In the above discussion we presented OS-POD by means of the spatial correlation operator $\mathcal{R}$. There is an equivalent formulation based on a temporal correlation operator $\mathcal{K}$. For numerical realizations one can choose the formulation involving $\mathcal{R}$, respectively $\mathcal{K}$, depending on which of the two results in a descretized eigenvalue problem of smaller dimension.

To overcome the problem of unmodelled dynamics in the POD-basis it was proposed earlier $[1,19]$ to update the basis after a solution to $\left(\mathbf{P}^{\ell}\right)$, to recompute the solution to $\left(\mathbf{P}^{\ell}\right)$ with this new basis, and to possibly iterate. In [2] this updating procedure was combined with a trust region strategy which determines whether at the end of an optimization step an update of the POD-basis should be performed. The main difference between the procedure just described and $\left(\mathbf{P}_{\mathrm{OS}-\mathrm{POD}}^{\ell}\right)$ lies in the fact that the former updates the basis according to the optimal control obtained from $\left(\mathbf{P}^{\ell}\right)$ whereas the update of the POD-basis following ( $\mathbf{P}_{\text {OS-POD }}^{\ell}$ ) respects the goal of minimizing $J$. On a computational level, updating the basis at the end of a ( $\mathbf{P}_{\text {OS-POD }}^{\ell}$ ) solution step requires one forward solve of the full system, whereas a gradient step for $\left(\mathbf{P}^{\ell}\right)$ for $\mathbf{z}_{2}$ requires one forward and one backwards solve of the full system.

Let us briefly point to some of the literature on model reduction based on POD. These citations are only of exemplary nature. We refer to $[5,22]$ as two of the early works on POD much of which is in the context of fluid dynamics. For optimal control and inverse problems POD was used for a wide variety of diffusion dominated systems, for instance in $[4,12,16,23]$. In [14] optimal closed loop control for Burgers equation is based on POD reduction of the state equation to solve the Hamilton-Jacobi-Bellman equation. For linear systems POD is closely related to balanced truncation, we refer to $[8,15,18,21,28]$. Reduced basis methods also utilize states of the underlying system to generate a basis but, unlike POD, they do not involve an orthogonalization step, see [11] in the context of optimal control, for example. While in the present paper we fix the dimension of the POD-subspace, approaches to the problem of convergence of the reduced order dynamical system to the infinite dimensional system as $\ell \rightarrow \infty$ are proposed in $[9,10,13]$. This question is nonstandard, since one cannot rely on approximation theory results as in the case of finite elements for the convergence of elements in $X^{\ell}$ to those in $X$. A difficulty related to POD-based model reduction may result from the evaluation of the nonlinearity on the POD subspace [3]. In this respect, reduced-basis elements $[7,20]$ offer an interesting alternative. 
The contents of the following sections is the following. In Section 2 the precise problem statement is given. Existence of an optimal solution and associated Lagrange multipliers is claimed and a first order optimality system is derived. The proofs to the claims in Section 2 are given in Section 3. Section 4 contains selected numerical results which demonstrate the feasibility of the proposed methodology.

\section{EXISTENCE FOR AUGMENTED SYSTEMS AND OPTIMALITY CONDITION}

The dynamical system under consider is of the form

$$
\frac{\mathrm{d}}{\mathrm{d} t}\langle y(t), \varphi\rangle_{H}+a(y(t), \varphi)+\langle\mathcal{N}(y(t)), \varphi\rangle_{V^{*}, V}=\sum_{k=1}^{m} u_{k}(t)\left\langle b_{k}, \varphi\right\rangle_{H}
$$

for allmost all $t \in(0, T]$ and

$$
\langle y(0), \varphi\rangle_{H}=\left\langle y_{0}, \varphi\right\rangle_{H} \quad \text { for all } \varphi \in V
$$

with the following specifications holding throughout

- $T>0, V$ and $H$ are separable real Hilbert spaces, with $V$ dense and compact in $H$, and $V \subset H=$ $H^{*} \subset V^{*}$ a Gelfand triple;

- $a: V \times V \rightarrow \mathbb{R}$ is a symmetric bilinear form satisfying $a(\varphi, \varphi) \geq \alpha\|\varphi\|_{V}^{2}$ for some $\alpha>0$ independent of $\varphi$;

- $\mathcal{N}: V \rightarrow V^{*}$ is a twice continuously Fréchet-differentiable operator;

- the control shape functions $b_{k}$ are chosen in $H$ with control intensities $u \in L^{2}\left(0, T ; \mathbb{R}^{m}\right)$;

- $y_{0} \in V$.

We associate with $a$ the isomorphism $\mathcal{A}: V \rightarrow V^{*}$, which can alternatively be considered as linear unbounded selfadjoint operator in $H$ with domain $D(\mathcal{A})=\{\varphi \in V: \mathcal{A} \varphi \in H\}$. Defining $\mathcal{B}: \mathbb{R}^{m} \rightarrow H$ by $\mathcal{B}(v)=\sum_{k=1}^{m} v_{k} b_{k}$ we can express $(2.1)$ in operator form as

$$
\left\{\begin{array}{l}
\frac{\mathrm{d}}{\mathrm{d} t} y(t)+\mathcal{A} y(t)+\mathcal{N}(y(t))=\mathcal{B}(u(t)) \quad \text { for } t \in(0, T] \\
y(0)=y_{0}
\end{array}\right.
$$

Further assumptions are necessary for the nonlinearity $\mathcal{N}$. We choose conditions which are satisfied for nonlinearities of Navier-Stokes type, see [24], Chapter III, for example.

$$
\left\{\begin{array}{l}
\text { For every } u \in L^{2}\left(\mathbb{R}^{m}\right) \text { there exists a unique solution } \\
y=y(u) \in L^{2}(D(\mathcal{A})) \cap H^{1}(V) \text { and moreover } \\
\text { there exists a continuous function } c_{1}: \mathbb{R} \rightarrow \mathbb{R} \text { such that } \\
\|y(u)\|_{L^{2}(D(\mathcal{A})) \cap H^{1}(V)} \leq c_{1}\left(\|u\|_{L^{2}\left(\mathbb{R}^{m}\right)}\right) \text { for all } u \in L^{2}\left(\mathbb{R}^{m}\right) .
\end{array}\right.
$$

Here and throughout we shall abbreviate $L^{2}(0, T ; Y)$ by $L^{2}(Y)$, and analogously for $H^{1}(0, T ; Y)$ and $C([0, T] ; Y)$. We further require the assumptions

$$
\left\{\begin{array}{l}
\text { there exist real constants } c_{2} \text { and } c_{3} \text { such that } \\
-\langle\mathcal{N}(\psi), \psi\rangle_{V^{*}, V} \leq \frac{\alpha}{2}\|\psi\|_{V}^{2}+c_{2}\|\psi\|_{H}^{2}+c_{3} \text { for all } \psi \in V \text { and } \\
\mathcal{N} \text { maps bounded sets in } V \text { to bounded sets in } V^{*}
\end{array}\right.
$$

and

$$
D(\mathcal{A}) \text { embeds compactly into } V \text {. }
$$


For the Navier-Stokes nonlinearity, $(\mathbf{H 2})$ is satisfied with $c_{2}=c_{3}=0$. We consider an optimal control problem of tracking type. Different cost functionals could be treated quite analogously.

$$
\left\{\begin{array}{l}
\min J(y, u)=\min \frac{\beta}{2} \int_{0}^{T}\|y(t)-z(t)\|_{H}^{2} \mathrm{~d} t+\frac{1}{2} \int_{0}^{T} u(t)^{T} \mathbf{R} u(t) \mathrm{d} t \\
\text { subject to } u \in L^{2}\left(\mathbb{R}^{m}\right) \text { and }(2.1),
\end{array}\right.
$$

where $\beta>0, z \in L^{2}(H)$, and $\mathbf{R} \in \mathbb{R}^{m \times m}$ is positive definite and symmetric. To denote the reduced cost functional we write $\hat{J}(u)=J(y(u), u)$, with $y(u)$ the solution to (2.1) for given $u$. With (H1) holding it is standard to argue existence of a solution $\left(y^{*}, u^{*}\right)=\left(y\left(u^{*}\right), u^{*}\right)$ to $(\mathrm{P})$. It also follows from proof to Theorem 2.1 below.

To define the POD reduction with basis $\left\{\psi_{i}\right\}_{i=1}^{\ell}$ let

$$
X=H \quad \text { or } \quad X=V
$$

and for $y \in L^{2}(X)$ let $\mathcal{R}: X \rightarrow X$ be given by

$$
\mathcal{R} \psi=\int_{0}^{T}\langle y(t), \psi\rangle_{X} y(t) \mathrm{d} t \quad \text { for } \psi \in X .
$$

Clearly $\mathcal{R}$ is a bounded, nonnegative, selfadjoint operator which can be expressed as

$$
\mathcal{R}=\mathcal{Y Y}^{*}
$$

where $\mathcal{Y}: L^{2}(\mathbb{R}) \rightarrow X$ is defined by

$$
\mathcal{Y} v=\int_{0}^{T} v(t) y(t, \cdot) \mathrm{d} t \quad \text { for } v \in L^{2}(\mathbb{R}),
$$

and the adjoint $\mathcal{Y}^{*}: X \rightarrow L^{2}(\mathbb{R})$ is given by

$$
\mathcal{Y}^{*} \psi=\langle y(t, \cdot), \psi(\cdot)\rangle_{X} \quad \text { for } \psi \in X
$$

We shall also utilize the operator $\mathcal{K}: L^{2}(\mathbb{R}) \rightarrow L^{2}(\mathbb{R})$ defined by

$$
\mathcal{K}=\mathcal{Y}^{*} \mathcal{Y}
$$

or explicitly

$$
(\mathcal{K} v)(t)=\int_{0}^{T}\langle y(t, \cdot), y(s, \cdot)\rangle_{X} v(s) \mathrm{d} s \quad \text { for } v \in L^{2}(\mathbb{R}) .
$$

Proposition 2.1. Let $y \in L^{2}(X)$. Then the operator $\mathcal{K}$ is compact. Moreover, except for possibly $0, \mathcal{K}$ and $\mathcal{R}$ possess the same eigenvalues which are positive with identical multiplicities and $\psi$ is eigenvector of $\mathcal{R}$ if and only if $\mathcal{Y}^{*} \psi=\langle y(t, \cdot), \psi\rangle_{X}$ is an eigenvector of $\mathcal{K}$.

Proof. Since the kernel of $\mathcal{K}$ is square integrable on $(0, T) \times(0, T)$ the integral operator $\mathcal{K}$ is Hilbert-Schmidt and therefore compact. From $\mathcal{K}=\mathcal{Y}^{*} \mathcal{Y}$ we deduce that $\mathcal{K}$ is selfadjoint and nonnegative. Its positive spectral values are necessarily eigenvalues with finite multiplicity and their only possible accumulation point is 0 . If $\varphi$ is an eigenvector of $\mathcal{K}$ with eigenvalue $\lambda>0$ then $\mathcal{Y}^{*} \mathcal{Y} \varphi=\lambda \varphi$ and thus $\mathcal{Y} \varphi$ is an eigenvector of $\mathcal{R}$. Analogously, if $\psi$ is an eigenvector of $\mathcal{R}$ with eigenvalue $\lambda \neq 0$ then $\mathcal{Y}^{*} \psi$ is an eigenvector of $\mathcal{K}$. Let $\lambda \neq 0$ be an eigenvalue of $\mathcal{K}$ and 
let ker $\{\mathcal{K}-\lambda I\}=\operatorname{span}\left\{\varphi_{i}\right\}_{i=1}^{r}$ with $\left\{\varphi_{i}\right\}_{i=1}^{r}$ linearly independent. Then $\left\{\mathcal{Y} \varphi_{i}\right\}_{i=1}^{r}$ are linearly independent. If not, then there exist scalars $\alpha_{i}, 1 \leq i \leq r$, with $\prod_{i=1}^{r} \alpha_{i} \neq 0$ such that $\sum_{i=1}^{r} \alpha_{i} \mathcal{Y} \varphi_{i}=0$. This implies

$$
0=\mathcal{Y}^{*}\left(\sum_{i=1}^{r} \alpha_{i} \mathcal{Y} \varphi_{i}\right)=\sum_{i=1}^{r} \alpha_{i} \mathcal{K} \varphi_{i}=\lambda \sum_{i=1}^{r} \alpha_{i} \varphi_{i}
$$

which is impossible, since $\lambda>0$ and $\left\{\varphi_{i}\right\}_{i=1}^{r}$ are linearly independent. Hence $\operatorname{dim} \operatorname{ker}\{\mathcal{R}-\lambda I\} \geq \operatorname{dim} \operatorname{ker}\{\mathcal{K}-$ $\alpha I\}$. The converse inequality follows analogously and hence $\operatorname{dim} \operatorname{ker}\{\mathcal{R}-\lambda I\}=\operatorname{dim} \operatorname{ker}\{\mathcal{K}-\lambda I\}$.

We shall utilize POD bases $\left\{\psi_{i}(y)\right\}_{i=1}^{\ell}$ with respect to $X=H$ or $X=V$ satisfying $\lambda_{1} \geq \lambda_{2} \geq \ldots \geq \lambda_{\ell}>0$, and

$$
\begin{aligned}
\mathcal{R}(y) \psi_{i}=\int_{0}^{T}\left\langle y(t, \cdot), \psi_{i}\right\rangle_{X} y(t) \mathrm{d} t & =\lambda_{i} \psi_{i} & & \text { for } i=1, \ldots, \ell, \\
\left\langle\psi_{i}, \psi_{j}\right\rangle_{X} & =\delta_{i j} & & \text { for } i, j=1, \ldots, \ell .
\end{aligned}
$$

The POD-subspaces are denoted by

$$
V^{\ell}=\operatorname{span}\left\{\psi_{1}, \ldots, \psi_{\ell}\right\}
$$

Note that $V^{\ell}$ depends on $y$. In this paper the POD-subspaces are generated by trajectories $y$ which arise as controlled trajectories of (2.1). We shall require the following condition

$$
\min \left\{\lambda_{\ell}(\mathcal{R}(y)) \mid y \text { solves }(2.1) \text { with } u \in L^{2}\left(\mathbb{R}^{m}\right)\right\}>0 .
$$

Note that $\psi_{i} \in V$ also for $X=H$. This follows from (2.3a) using that $y \in L^{2}(V)$. Moreover, $\psi_{i} \in D(\mathcal{A})$ for $y \in L^{2}(D(\mathcal{A}))$.

To obtain the POD-Galerkin approximation to (2.1) we make the ansatz

$$
y^{\ell}(t)=\sum_{j=1}^{\ell} x_{j}(t) \psi_{j}
$$

replace $y$ by $y^{\ell}$ in (2.1), take inner products in $H$ with respect to $\left\{\psi_{i}\right\}_{i=1}^{\ell}$ and obtain the system of ordinary differential equations in $\mathbb{R}^{\ell}$

$$
\left\{\begin{array}{l}
E(\psi) \dot{x}(t)+A(\psi) x(t)+\mathfrak{N}(x(t), \psi)=B(\psi) u(t) \quad \text { for } \quad t \in(0, T] \\
E(\psi) x(0)=x_{0}(\psi)
\end{array}\right.
$$

Here $E: X^{\ell} \times X^{\ell} \rightarrow \mathbb{R}^{\ell \times \ell}$ with $X^{\ell}=\bigotimes_{i=1}^{\ell} X$ is defined by

$$
E_{i j}(\varphi, \phi)=\left\langle\varphi_{i}, \phi_{j}\right\rangle_{H} \text { and } E(\varphi)=E(\varphi, \varphi),
$$

$A: X^{\ell} \times X^{\ell} \rightarrow \mathbb{R}^{\ell \times \ell}$ is defined by

$$
A_{i j}(\varphi, \phi)=a\left(\varphi_{i}, \phi_{j}\right) \text { and } A(\varphi)=A(\varphi, \varphi),
$$

$B: X^{\ell} \rightarrow \mathbb{R}^{\ell \times m}$ and $x_{0}: X^{\ell} \rightarrow \mathbb{R}^{\ell}$ are given by

$$
B_{i j}(\varphi)=\left\langle\varphi_{i}, b_{j}\right\rangle_{H}, \quad x_{0, i}(\varphi)=\left\langle y_{0}, \varphi_{i}\right\rangle_{H},
$$


and the nonlinearity $\mathfrak{N}: \mathbb{R}^{\ell} \times X^{\ell} \times X^{\ell} \rightarrow \mathbb{R}^{\ell}$ by

$$
\mathfrak{N}_{i}(x, \psi, \varphi)=\left\langle\mathcal{N}\left(\sum_{j=1}^{\ell} x_{j} \psi_{j}\right), \varphi_{i}\right\rangle_{V^{*}, V} \text { with } \mathfrak{N}(x, \varphi)=\mathfrak{N}(x, \varphi, \varphi) .
$$

Discretizing the cost function in the same manner we obtain

$$
\begin{aligned}
J^{\ell}(x, \psi, u)= & \frac{\beta}{2} \int_{0}^{T}\left(x(t)^{T}\left(E(\psi) x(t)-2 z^{\ell}(t, \psi)\right)+\|z(t)\|_{H}^{2}\right) \mathrm{d} t \\
& +\frac{1}{2} \int_{0}^{T} u^{T}(t) \mathbf{R} u(t) \mathrm{d} t,
\end{aligned}
$$

where $z^{\ell}:(0, T) \times X^{\ell} \rightarrow \mathbb{R}^{\ell}$ is given by

$$
z^{\ell}(t, \varphi)_{i}=\left\langle z(t), \varphi_{i}\right\rangle_{H}
$$

and $J^{\ell}: L^{2}\left(\mathbb{R}^{\ell}\right) \times X^{\ell} \times L^{2}\left(\mathbb{R}^{m}\right) \rightarrow \mathbb{R}^{+}$.

We are now prepared to specify the POD-Galerkin reduced optimal control problem augmented with the POD-generation criteria:

$$
\left\{\begin{array}{l}
\min J^{\ell}(x, \psi, u) \quad \text { over } \quad(x, \psi, u) \in L^{2}\left(\mathbb{R}^{\ell}\right) \times X^{\ell} \times L^{2}\left(\mathbb{R}^{m}\right), \\
\text { subject to } \\
E(\psi) \dot{x}(t)+A(\psi) x(t)+\mathfrak{N}(x(t), \psi)=B(\psi) u(t) \quad \text { for } t \in(0, T] \\
E(\psi) x(0)=x_{0}(\psi), \\
\frac{\mathrm{d}}{\mathrm{d} t} y(t)+\mathcal{A} y(t)+\mathcal{N}(y(t))=\mathcal{B}(u(t)) \quad \text { for } t \in(0, T] \\
y(0)=y_{0}, \\
\mathcal{R}(y) \psi_{i}=\lambda_{i} \psi_{i} \quad \text { for } i=1, \ldots, \ell \\
\left\langle\psi_{i}, \psi_{j}\right\rangle_{X}=\delta_{i j} \quad \text { for } i, j=1, \ldots, \ell
\end{array}\right.
$$

As discussed in Section 1, if the POD-eigenvalue problem is solved at a reference trajectory $y(\bar{u})$ corresponding to a fixed reference control $\bar{u}$, this results in the last four equations from ( $\left.\mathrm{P}_{\mathrm{OS}-\mathrm{POD}}\right)$. The remaining optimization is the standard one in the POD-Galerkin optimal control approach.

Theorem 2.2. If (H1)-(H4) hold, then ( $\left.\mathrm{P}_{\mathrm{OS}-\mathrm{POD}}\right)$ admits a (global) solution $\left(x^{*}, \psi^{*}, u^{*}\right) \in W^{1,2}\left(\mathbb{R}^{\ell}\right) \times X^{\ell} \times$ $L^{2}\left(\mathbb{R}^{m}\right)$ with $\left(\lambda^{*}, y^{*}\right) \in \mathbb{R}^{m} \times\left(L^{2}(D(\mathcal{A})) \cap W^{1,2}(V)\right)$ and $y^{*}=y\left(u^{*}\right)$.

The proof of this theorem and the following ones are given in the Appendix.

We proceed by deriving an optimality system. For this purpose we assume that the eigenvalues of $\mathcal{R}\left(y^{*}\right)$ with $y^{*}=y\left(u^{*}\right)$ are distinct. If this is not the case then in the following results we have to keep the orthonormality condition on the subspace corresponding to a multiple eigenvalue as explicit constraints. For $\lambda_{i} \neq \lambda_{j}$ we have $\left\langle\psi_{i}, \psi_{j}\right\rangle_{X}=0$ since $\mathcal{R}$ is selfadjoint. Therefore $(2.3 \mathrm{~b})$ will be replaced by $\left\|\psi_{i}^{*}\right\|_{X}=1$ for $i=1, \ldots, \ell$.

Henceforth the state and the control variables are considered in the space

$$
Z=H^{1}\left(\mathbb{R}^{\ell}\right) \times W(0, T) \times X^{\ell} \times \mathbb{R}^{\ell} \times L^{2}\left(\mathbb{R}^{m}\right),
$$

where $W(0, T)=L^{2}(V) \cap H^{1}\left(V^{*}\right)$ and the generic element of $Z$ is denoted by $\mathbf{z}=(x, y, \psi, \lambda, u)$. We utilize adjoint variables form the space

$$
\Xi=L^{2}\left(\mathbb{R}^{\ell}\right) \times \mathbb{R}^{\ell} \times L^{2}(V) \times H \times X^{\ell} \times \mathbb{R}^{\ell}
$$


with generic element

$$
\xi=\left(q, q_{0}, p, p_{0}, \mu, \eta\right)
$$

To describe the six equality constraints in $\left(\mathrm{P}_{\mathrm{OS}-\mathrm{POD}}^{\ell}\right)$ we introduce

$$
\begin{array}{ll}
e_{1}: Z \rightarrow L^{2}\left(\mathbb{R}^{\ell}\right), & e_{2}: Z \rightarrow \mathbb{R}^{\ell}, \\
e_{3}: Z \rightarrow L^{2}\left(V^{*}\right), & e_{4}: Z \rightarrow H, \\
e_{5}: Z \rightarrow X^{\ell}, & e_{6}: Z \rightarrow \mathbb{R}^{\ell}
\end{array}
$$

by

$$
\begin{aligned}
& e_{1}(\mathbf{z})=E(\psi) \dot{x}+A(\psi) x+\mathfrak{N}(x(\cdot), \psi)-B(\psi) u \\
& e_{2}(\mathbf{z})=E(\psi) x(0)-x_{0}(\psi) \\
& e_{3}(\mathbf{z})=y_{t}+\mathcal{A} y+\mathcal{N}(y(\cdot))-\mathcal{B} u \\
& e_{4}(\mathbf{z})=y(0)-y_{0} \\
& e_{5}(\mathbf{z})=\left(\left(\mathcal{R}-\lambda_{1} I\right) \psi_{1}, \ldots,\left(\mathcal{R}-\lambda_{l} I\right) \psi_{\ell}\right) \\
& e_{6}(\mathbf{z})=\left(\left\|\psi_{1}\right\|_{X}^{2}-1, \ldots,\left\|\psi_{\ell}\right\|_{X}^{2}-1\right)
\end{aligned}
$$

To obtain a first-order necessary optimality condition we introduce the Lagrange functional $\mathcal{L}: Z \times \Xi \rightarrow \mathbb{R}$ by

$$
\begin{aligned}
\mathcal{L}(\mathbf{z}, \xi)= & J^{\ell}(x, \psi, u)+\left\langle e_{1}(\mathbf{z}), q\right\rangle_{L^{2}\left(\mathbb{R}^{\ell}\right)}+\left\langle e_{2}(\mathbf{z}), q_{0}\right\rangle_{\mathbb{R}^{\ell}} \\
& +\left\langle e_{3}(\mathbf{z}), p\right\rangle_{L^{2}\left(V^{*}\right), L^{2}(V)}+\left\langle e_{4}(\mathbf{z}), p_{0}\right\rangle_{H}+\left\langle e_{5}(\mathbf{z}), \mu\right\rangle_{X^{\ell}}+\left\langle e_{6}(\mathbf{z}), \eta\right\rangle_{\mathbb{R}^{\ell}}
\end{aligned}
$$

Subsequently we derive the system which arises from setting $\mathcal{L}_{z}(\mathbf{z}, \xi)=0$. Then we argue existence of $\xi^{*}$ such that $\mathcal{L}_{z}\left(\mathbf{z}^{*}, \xi^{*}\right)=0$ and finally we prove that $\mathcal{L}_{z}\left(\mathbf{z}^{*}, \xi^{*}\right)=0$ is a necessary optimality condition. Setting $\mathcal{L}_{x}(\mathrm{z}, \xi)=0$ we find

$$
\left\{\begin{array}{l}
-E(\psi) \dot{q}(t)+\left(A(\psi)+\mathfrak{N}_{x}^{T}(x(t), \psi)\right) q(t)=\beta(z(t, \psi)-E(\psi) x(t)) \\
q(T)=0
\end{array}\right.
$$

and

$$
q_{0}=q(0)
$$

which is the common adjoint equation in the context of POD-Galerkin optimal control. To compute $\mathcal{L}_{y}(z, \xi)$ we first consider the term $e_{5}(\mathrm{z})$ and denote by $\mathcal{I}: X^{*} \rightarrow X$ the canonical Riesz isomorphism, which is the identity operator in case of $X=H$. We find

$$
\begin{aligned}
\frac{\partial}{\partial y}\left(\left\langle\left(\mathcal{R}-\lambda_{i} I\right) \psi_{i}, \mu_{i}\right\rangle_{X}\right) \delta y & =\int_{0}^{T}\left\langle\left\langle y(t), \psi_{i}\right\rangle_{X} \mu_{i}+\left\langle y(t), \mu_{i}\right\rangle_{X} \psi_{i}, \delta y(t)\right\rangle_{X} \mathrm{~d} t \\
& =\int_{0}^{T}\left\langle\left\langle y(t), \psi_{i}\right\rangle_{X} \mathcal{I}^{-1} \mu_{i}+\left\langle y(t), \mu_{i}\right\rangle_{X} \mathcal{I}^{-1} \psi_{i}, \delta y(t)\right\rangle_{V^{*}, V} \mathrm{~d} t
\end{aligned}
$$

for $i \in\{1, \ldots, \ell\}$. Thus, $\mathcal{L}_{y}(\mathbf{z}, \xi)=0$ implies

$$
\left\{\begin{aligned}
-\dot{p}(t) & +\mathcal{A} p(t)+\mathcal{N}^{\prime}(y(t))^{*} p(t) \\
= & \sum_{i=1}^{\ell}\left\langle y(t), \mu_{i}\right\rangle_{X} \mathcal{I}^{-1} \psi_{i}+\left\langle y(t), \psi_{i}\right\rangle_{X} \mathcal{I}^{-1} \mu_{i} \\
p(T) & =0
\end{aligned}\right.
$$


and

$$
p_{0}=p(0)
$$

i.e., the adjoint equation on the level of the original systems couples with the POD-reduction through the linearization of the kernel of the correlation operator. From $\mathcal{L}_{\lambda}(\mathbf{z}, \xi)=0$ we derive

$$
\left\langle\psi_{i}, \mu_{i}\right\rangle_{X}=0 \text { for } 1, \ldots, \ell
$$

and $\mathcal{L}_{u}(\mathbf{z}, \xi)=0$ implies

$$
\mathbf{R} u(t)=B(\psi)^{T} q(t)+\mathcal{B}^{*} p(t)
$$

which is the optimality condition. Note, if we were to only consider the POD-reduced optimal control problem then (2.11) would be replaced by

$$
\mathbf{R} u(t)=B(\psi)^{T} q(t)
$$

Finally we turn to $\mathcal{L}_{\psi}(\mathbf{z}, \xi)=0$, which involves the terms $e_{1}, e_{2}, e_{5}$ and $e_{6}$ as well as $J^{\ell}$. For any $\delta \psi \in X^{\ell}$ we have

$$
\begin{aligned}
0=\mathcal{L}_{\psi}(\mathbf{z}, \xi) \delta \psi= & \langle\mathcal{G}(x, \psi, u, q), \delta \psi\rangle_{\left(X^{\ell}\right)^{*}, X^{\ell}}+\sum_{i=1}^{\ell}\left\langle\left(\mathcal{R}-\lambda_{i} I\right) \delta \psi_{i}, \mu_{i}\right\rangle_{X} \\
& +2 \sum_{i=1}^{\ell}\left\langle\delta \psi_{i}, \psi_{i}\right\rangle_{X} \eta_{i} .
\end{aligned}
$$

Here, $\mathcal{G}: H^{1}\left(\mathbb{R}^{\ell}\right) \times X^{\ell} \times L^{2}\left(\mathbb{R}^{m}\right) \times H^{1}\left(\mathbb{R}^{\ell}\right) \rightarrow\left(X^{\ell}\right)^{*}$ is given by

$$
\begin{aligned}
\langle\mathcal{G}(x, \psi, u, q), \delta \psi\rangle_{\left(X^{\ell}\right)^{*}, X^{\ell}}= & \beta \\
& \int_{0}^{T} x^{T}(E(\psi, \delta \psi) x-z(\cdot, \delta \psi)) \mathrm{d} t \\
& +\int_{0}^{T} q^{T}[(E(\psi, \delta \psi)+E(\delta \psi, \psi)) \dot{x}+(A(\psi, \delta \psi)+A(\delta \psi, \psi)) x] \mathrm{d} t \\
& +\int_{0}^{T} q^{T}\left[\mathfrak{N}_{\psi}(x, \psi) \delta \psi-B(\delta \psi) u\right] \mathrm{d} t+q(0)^{T}(E(\psi, \delta \psi)+E(\delta \psi, \psi)) x(u)
\end{aligned}
$$

Note that $\mathcal{G}$ can also be expressed as

$$
\langle\mathcal{G}(x, \psi, u, q), \delta \psi\rangle_{\left(X^{\ell}\right)^{*}, X^{\ell}}=\sum_{k=1}^{\ell}\left\langle\mathcal{G}_{k}(x, \psi, u, q), \delta \psi_{k}\right\rangle_{X^{*}, X},
$$

where

and

$$
\left\langle\mathcal{G}_{k}(x, \psi, u, q), \delta \psi_{k}\right\rangle_{X^{*}, X}=\left\langle\mathcal{G}(x, \psi, u, q), \widehat{\delta \psi}_{k}\right\rangle_{\left(X^{\ell}\right)^{*}, X^{\ell}}
$$

Then we have

$$
\left(\widehat{\delta \psi}_{k}\right)_{i}=0 \quad \text { if } \quad i \neq k, \quad\left(\widehat{\delta \psi}_{k}\right)_{k}=\delta \psi_{k}
$$

$$
\left(\mathcal{R}-\lambda_{i} I\right) \mu_{i}+2 \eta_{i} \psi_{i}=-\mathcal{I} \mathcal{G}_{i}(x, \psi, u, q) \quad \text { for } i=1, \ldots, \ell
$$

By (2.10) this implies

and

$$
\eta_{i}=-\frac{1}{2}\left\langle\mathcal{G}_{i}(x, \psi, u, q), \psi_{i}\right\rangle_{X^{*}, X}
$$

$$
\left(\mathcal{R}-\lambda_{i} I\right) \mu_{i}=-2 \eta_{i} \psi_{i}-\mathcal{I} \mathcal{G}_{i}(x, \psi, u, q) \quad \text { for } i=1, \ldots, \ell
$$


Since the right-hand side of this equation is orthogonal to the kernel of $\mathcal{R}-\lambda_{i} I$ it admits a unique solution $\mu_{i}$. The explicit form of $\mathcal{G}_{i}$ is found to be

$$
\begin{aligned}
\mathcal{G}_{i}(x, \psi, u, q)= & \int_{0}^{T}\left(x_{i}\left(\sum_{j=1}^{\ell} x_{j} \psi_{j}-z\right)+q_{i} \sum_{j=1}^{\ell} \dot{x}_{j} \psi_{j}+\dot{x}_{j} \sum_{j=1}^{\ell} q_{j} \psi_{j}\right) \mathrm{d} t \\
& +\int_{0}^{T}\left(q_{i} \sum_{j=1}^{\ell} x_{j} \mathcal{A} \psi_{j}+x_{i} \sum_{j=1}^{\ell} q_{j} \mathcal{A} \psi_{j}-q_{i} \sum_{k=1}^{m} b_{k} u_{k}\right) \mathrm{d} t \\
& +\sum_{j=1}^{\ell}\left(x_{j}(0) \psi_{j} q_{i}(0)+x_{i}(0) \psi_{j} q_{j}(0)\right)-y_{0} q_{i}(0) \\
& +\mathcal{N}\left(\sum_{k=1}^{\ell} x_{k} \psi_{k}\right) q_{i}+x_{i} \sum_{j=1}^{\ell} \mathcal{N}^{\prime}\left(\sum_{k=1}^{\ell} x_{k} \psi_{k}\right)^{*} q_{j} \psi_{j} .
\end{aligned}
$$

Theorem 2.3. Let (H1) $-(\mathbf{H} 4)$ hold and let

$$
\mathrm{z}=(x, y, \psi, \lambda, u) \in W^{1,2}\left(\mathbb{R}^{\ell}\right) \times W^{1,2}(V) \times X^{\ell} \times \mathbb{R}^{m} \times L^{2}\left(\mathbb{R}^{m}\right)
$$

denote a solution to $\left(\mathrm{P}_{\mathrm{OS}-\mathrm{POD}}^{\ell}\right)$. Assume that the eigenvalues of $\mathcal{R}(y)$ are distinct and that

$$
\frac{\mathrm{d}}{\mathrm{d} t} v+\mathcal{A} v+\mathcal{N}^{\prime}(y(t)) v-\mathcal{B} \tilde{u}=w \text { for } t \in(0, T] \quad \text { and } \quad v(0)=v_{0}
$$

admits a solution $(v, \tilde{u}) \in W(0, T) \times L^{2}\left(\mathbb{R}^{m}\right)$ for every $\left(w, v_{0}\right) \in L^{2}\left(V^{*}\right) \times H$. Then there exist $(q, p, \mu, \eta) \in$ $L^{2}\left(\mathbb{R}^{\ell}\right) \times L^{2}(V) \times X^{\ell} \times \mathbb{R}^{\ell}$ such that the following optimality system holds:

$$
\begin{aligned}
& \left\{\begin{array}{l}
-E(\psi) \dot{q}(t)+\left(A(\psi)+\mathfrak{N}_{x}^{T}(x(t), \psi)\right) q(t)=-\beta(E(\psi) x(t)-z(t, \psi)), \\
q(T)=0
\end{array}\right. \\
& \left\{\begin{array}{c}
-\dot{p}(t)+\mathcal{A} p(t)+\mathcal{N}^{\prime}(y(t))^{*} p(t)= \\
\quad \sum_{i=1}^{\ell}\left\langle y(t), \mu_{i}\right\rangle_{X} \mathcal{I}^{-1} \psi_{i}+\left\langle y(t), \psi_{i}\right\rangle_{X} \mathcal{I}^{-1} \mu_{i}, \\
p(T)=0
\end{array}\right. \\
& \left\{\begin{array}{l}
\eta_{i}=-\frac{1}{2}\left\langle\mathcal{G}_{i}(x, \psi, u, q), \psi_{i}\right\rangle_{X^{*}, X} \\
\mu_{i}=-\left(\mathcal{R}-\lambda_{i} I\right)^{-1}\left[2 \eta_{i} \psi_{i}+\mathcal{I} \mathcal{G}_{i}(x, \psi, u, q)\right] \quad \text { for } i=1, \ldots, \ell,
\end{array}\right. \\
& \mathbf{R} u(t)=B^{T}(\psi) q(t)+\mathcal{B}^{*} p(t) .
\end{aligned}
$$

The following theorem addresses the situation when $\mathcal{K}$ is of smaller dimension than $\mathcal{R}$.

Theorem 2.4. Let $\mathrm{z}=(x, y, \psi, \lambda, u) \in Z$ denote a solution of ( $\left.\mathrm{P}_{\mathrm{OS}-\mathrm{POD}}\right)$ and let the assumptions of Theorem 2.3 hold. Then there exists $\left(q, p^{\mathcal{K}}, \mu^{\mathcal{K}}, \eta\right) \in L^{2}\left(\mathbb{R}^{\ell}\right) \times L^{2}(V) \times X^{\ell} \times \mathbb{R}^{\ell}$ satisfying the optimality system 
consisting of (2.16) and

$$
\begin{aligned}
& \left\{\begin{array}{r}
-\dot{p}^{\mathcal{K}}(t)+\mathcal{A} p^{\mathcal{K}}(t)+\mathcal{N}^{\prime}(y(t))^{*} p^{\mathcal{K}}(t)= \\
\ell \\
\sum_{i=1}^{\ell} \int_{0}^{T} 2 \mathcal{I}^{-1} y(s, \cdot) \mu_{i}^{\mathcal{K}}(s) \mathrm{d} s \varphi_{i}(t) \\
+\sum_{i=1}^{\ell} \int_{0}^{T} \mathcal{I}^{-1} y(s, \cdot) \varphi_{i}(s) \mathrm{d} s \mu_{i}^{\mathcal{K}}(t)
\end{array}\right. \\
& \left\{\begin{array}{l}
\eta_{i}=-\frac{1}{2}\left\langle\mathcal{G}_{i}\left(x, \psi_{i}, u, q\right), \psi_{i}\right\rangle_{X^{*}, X} \\
\mu_{i}^{\mathcal{K}}=-\left(\mathcal{K}-\lambda_{i} I\right)^{-1}\left(\mathcal{K}^{-1} \tilde{\mathcal{G}}_{i}+2 \eta_{i} \varphi_{i}\right),
\end{array}\right. \\
& \mathbf{R} u(t)=B^{T}(\psi) q(t)+\mathcal{B}^{*} p^{\mathcal{K}}(t),
\end{aligned}
$$

where $\sqrt{\lambda_{i}} \psi_{i}=\mathcal{Y}^{*} \psi_{i}$ and $\tilde{\mathcal{G}}_{i}=\sqrt{\lambda_{i}} \mathcal{Y}^{*} \mathcal{I} \mathcal{G}_{i}\left(x, \psi_{i}, u, q\right)$

\section{NUMERICAL EXPERIMENTS}

In this section feasibility of the proposed methodology is demonstrated by means of optimal boundary control problems for the unsteady Burgers equation. We explain the numerical realization of OS-POD and present two test examples.

\subsection{Numerical strategy}

We consider the minimization problem

$$
\min J(y, u, v)=\frac{1}{2} \int_{0}^{T} \int_{0}^{1}|y-z|^{2} \mathrm{~d} x \mathrm{~d} t+\frac{\beta}{2} \int_{0}^{T}\left(|u|^{2}+|v|^{2}\right) \mathrm{d} t
$$

subject to

$$
\begin{aligned}
y_{t}-\nu y_{x x}+y y_{x} & =f & & \text { in } Q=(0, T) \times(0,1), \\
\nu y_{x}(\cdot, 0)+\sigma_{0} y(\cdot, 0) & =u & & \text { in }(0, T), \\
\nu y_{x}(\cdot, 1)+\sigma_{1} y(\cdot, 1) & =v & & \text { in }(0, T), \\
y(0, \cdot) & =y_{0} & & \text { in }(0,1) .
\end{aligned}
$$

In the context of Section 2 we set $H=L^{2}(0,1)$ and $V=H^{1}(0,1)$. We suppose $z \in L^{2}(0, T ; H), T>0$ and $\beta>0$. In (4.1b)-(4.1e) let $\nu>0, f \in L^{2}(0, T ; V), \sigma_{0} \in \mathbb{R}, \sigma_{1} \in \mathbb{R}$ and $y_{0} \in V$ be fixed.

Problem (4.1) admits at least one global optimal solution $x^{*}=\left(y^{*}, u^{*}, v^{*}\right)$; see e.g. [25-27]. Furthermore, there exist a unique associated Lagrange multiplier $p^{*} \in L^{2}(0, T ; V) \cap H^{1}\left(0, T ; V^{\prime}\right)$ satisfying

$$
\begin{aligned}
-p_{t}^{*}-\nu p_{x x}^{*}-y^{*} p_{x}^{*} & =-\left(y^{*}-z\right) & & \text { in } Q, \\
\nu p_{x}^{*}(\cdot, 0)+\left(\sigma_{0}+y^{*}(\cdot, 0)\right) p^{*}(\cdot, 0) & =0 & & \text { in }(0, T), \\
\nu p_{x}^{*}(\cdot, 1)+\left(\sigma_{1}+y^{*}(\cdot, 1)\right) p^{*}(\cdot, 1) & =0 & & \text { in }(0, T), \\
p^{*}(T, \cdot) & =0 & & \text { in }(0,1) .
\end{aligned}
$$

Let us recall how (4.1) was solved by a reduced-order approach in [26]. We shall use the same specifications for the discretisation and POD basis generation for the numerical results which follow. For given control inputs $u$ and $v(e . g ., u=v=0)$ a discrete solution $\left\{y_{h}^{j}\right\}_{j=0}^{m}$ to the unsteady Burgers equation (4.1b)-(4.1e) is computed 
by utilizing a finite element approximation for the spatial domain $(0,1)$ with piecewise linear ansatz functions and an implicit Euler method for time integration with an equidistant time grid $t_{j}=j T / m, 0 \leq j \leq m$. Instead of (1.1) we determine the POD basis by solving

$$
\left\{\begin{array}{l}
\min _{\left\{\psi_{i}\right\}_{i=1}^{\ell}} \sum_{j=0}^{m} \alpha_{j}\left\|y_{h}^{j}-\sum_{i=1}^{\ell}\left\langle y_{h}^{j}, \psi_{i}\right\rangle_{X} \psi_{i}\right\|_{X}^{2} \\
\text { subject to }\left\langle\psi_{i}, \psi_{j}\right\rangle_{X}=\delta_{i, j} \quad \text { for } 1 \leq i, j \leq \ell
\end{array}\right.
$$

where $y_{h}^{j}$ is the FE solution to (4.1b)-(4.1e) at time instance $t_{j}$ and the $\alpha_{j}$ 's denote trapezoidal weights given by $\alpha_{0}=\alpha_{m}=T /(2 m), \alpha_{j}=T / m$ for $j=1, \ldots, m-1$. The relationship between (1.1) and (4.3) was studied in [13]. If a POD basis is computed for the snapshot ensemble $\mathcal{V}=\operatorname{span}\left\{y_{h}^{0}, \ldots, y_{h}^{m}\right\} \subset V$, the POD-Galerkin projection $\left(\mathbf{P}^{\ell}\right)$ of $(4.1)$ is derived using $\ell$ POD basis functions. The corresponding low-dimensional optimal control problem was treated numerically by an SQP method resulting in a suboptimal solution $x^{\ell}=\left(y^{\ell}, u^{\ell}, v^{\ell}\right)$. Using $u=u^{\ell}$ and $v=v^{\ell}$ we solve (4.1b)-(4.1e) again and re-compute the POD basis. We iterated this strategy until the suboptimal controls converge within a given tolerance; see also [1,2].

The main difference between the previous approach and OS-POD results from the fact that in OS-POD the basis update respects the goal of minimizing $J$.

This structural benefit results in the difficulty that $\left(\mathrm{P}_{\mathrm{OS}-\mathrm{POD}}^{\ell}\right)$ not only involves the projected differential equation as a constraint, but also the eigenvalue problems $\mathcal{R}(y) \psi_{i}=\lambda_{i} \psi_{i}, 1 \leq i \leq \ell$. In the numerical realization these two constraints were not realized simultaneously but rather a splitting algorithm was used which alternatingly considered the constraints given by partial differential equation and the eigenvalue problems. The numerical strategy is presented next.

\section{Algorithm implemented}

(1) Choose an initial POD basis $\left\{\psi_{i}^{0}\right\}_{i=1}^{\ell}$ of $\operatorname{rank} \ell$ and set $n=0$.

(2) Compute the POD Galerkin approximation for (4.1).

(3) Solve $\left(\mathbf{P}^{\ell}\right)$ by a globalized SQP method for intermediate controls $(u, v)=\left(u_{+}^{\ell}, v_{+}^{\ell}\right)$ and the Lagrange multiplier $q$ solving $(2.16)$.

(4) Solve (4.1b)-(4.1e) with $(u, v)=\left(u_{+}^{\ell}, v_{+}^{\ell}\right)$, system (2.18) and the adjoint equation (2.17).

(5) Use (2.19) in a gradient step to obtain new control variables $\left(u_{n}^{\ell}, v_{n}^{\ell}\right)$.

(6) Determine a new POD basis $\left\{\psi_{i}^{n}\right\}_{i=1}^{\ell}$ of rank $\ell$ by solving $(4.1 \mathrm{~b})-(4.1 \mathrm{e})$ with $(u, v)=\left(u_{n}^{\ell}, v_{n}^{\ell}\right)$.

(7) Set $n=n+1$ and go back to back to (2).

Concerning (3) the SQP-iteration is carried out by $L U$-factorization and the SQP method is terminated at iteration level $k \geq 0$ provided the Lagrangian $\mathfrak{L}: H^{1}\left(\mathbb{R}^{\ell}\right) \times L^{2}(0, T) \times L^{2}(0, T) \times H^{1}\left(\mathbb{R}^{\ell}\right) \rightarrow \mathbb{R}$ associated with $\left(\mathbf{P}^{\ell}\right)$ satisfies

$$
\left\|\nabla \mathfrak{L}\left(x_{k}, u_{k}, v_{k}, q_{k}\right)\right\| \leq \varepsilon_{\text {rel }}^{n}\left\|\nabla \mathfrak{L}\left(x_{0}, u_{0}, v_{0}, q_{0}\right)\right\| \quad \text { for } k \geq 1
$$

with $\varepsilon^{0}=10^{-2}$ and $\varepsilon^{n}=\max \left\{10^{-4}, \min \left\{\|g\|_{L^{2}(0, T) \times L^{2}(0, T)}, \varepsilon^{n-1} / 10\right\}\right\}$ for $n \geq 1$, where $g=\mathbf{R} u-B(\psi)^{T} q-\mathcal{B}^{*} p$ denotes the OS-POD gradient (compare (2.11)). In the SQP iterations descent is achieved by using an $L_{1}$ merit function. We stop our algorithm provided

$$
\begin{aligned}
& -\varepsilon^{n}=10^{-4}, \\
& -\left\|\nabla \mathfrak{L}\left(x_{k}, u_{k}, v_{k}, q_{k}\right)\right\|<10^{-5} \text { and } \\
& -\|g\|_{L^{2}(0, T) \times L^{2}(0, T)}<\delta
\end{aligned}
$$

with $\delta=0.1$. In our numerical tests it turns out that decreasing $\delta$ does not essentially change the POD solutions, but rather leads to small variations in the POD basis functions.

A convergence analysis of this splitting method for solving $\left(\mathrm{P}_{\mathrm{OS}-\mathrm{POD}}^{\ell}\right)$ may be an interesting task which is, however, not within the scope of this paper. 

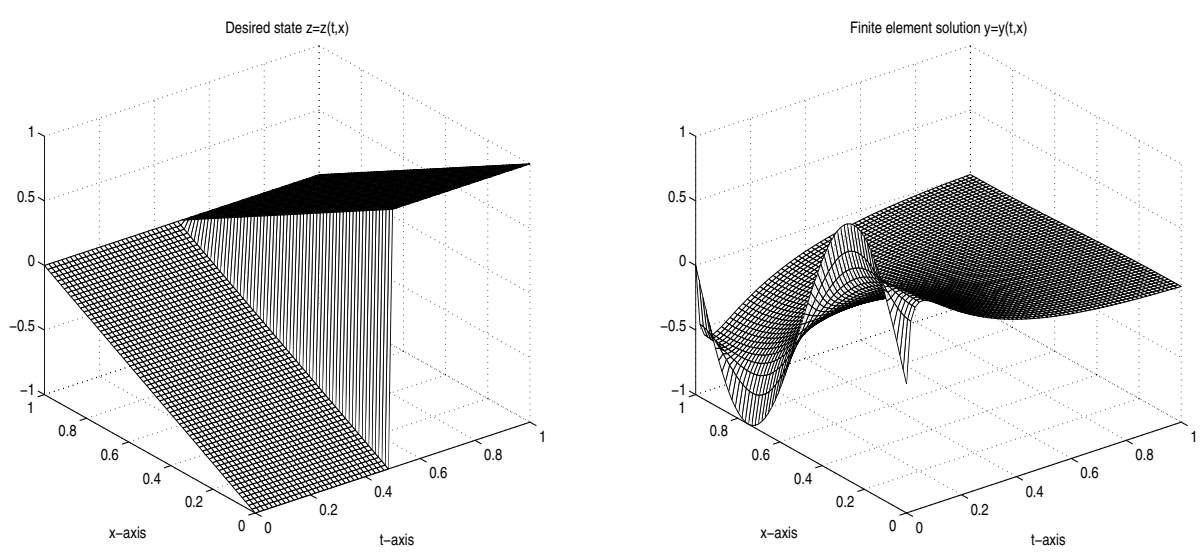

Figure 1. Run 1: desired state (left) and FE solution to the uncontrolled Burgers equation, i.e., $u=v=0$ (right).

\subsection{Test examples}

This section is devoted to numerical test examples illustrating the feasibility of the OS-POD approach. Comparisons will be made to the 'basic POD' approach which consists in solving $\left(\mathbf{P}^{\ell}\right)$ for a fixed control, here $u=v=0$, by an SQP-method applied to the optimality system, and to a FE-SQP approach, where the SQP iteration is carried out inexactly (using a preconditioned GMRES method) and it is terminated as soon as the same stopping criteria are hold as for the POD-SQP method (see [27] for more details).

Run 1. We consider (4.1) with $T=1, \beta=0.001, \nu=0.5, \sigma_{0}=0, \sigma_{1}=0.1, f(t, x)=\exp (-3 t) \sin (2 \pi x)$, $y_{0}(x)=\sin (2 \pi x), z(t, x)=x-1$ for $(t, x) \in[0, T / 2] \times[0,1]$ and $z(t, x)=1-x$ for $(t, x) \in(T / 2, T] \times[0,1] ;$ see Figure 1 (left plot). The FE solution to (4.1b)-(4.1e) is computed for the equidistant spatial grid $x_{i}=i / 256$ for $i=0, \ldots, 256$ and a semi-implicit Euler method on an equidistant time grid $t_{j}=j T / m$ for $j=0, \ldots, 256$, where the linear term $y_{t}-\nu y_{x x}$ is treated implicitly and the nonlinear term $y y_{x}$ explicitly in $y$, but implicitly in $y_{x}$. The corresponding FE solution for $u=v=0$ is presented in Figure 1 (right plot). The discrete solution is plotted on a four times coarser mesh than the computational grid.

Our first curiosity centers on the question whether the POD-basis is significantly changed during the OSPOD iterations. In this respect note that the solution to the uncontrolled Burgers equation with decay in the forcing as is the case for this example decays towards 0 as $t \rightarrow \infty$. Moreover there is transport in the direction of increasing (decreasing) $x$ where $y \geq 0(\leq 0)$ due to the convection term. The desired state $z$ does not share these properties so that the optimally controlled trajectory can be expected to be significantly different from the uncontrolled one, which is depicted in Figure 1 (right plot). Accordingly the basis elements corresponding to the uncontrolled and the optimally controlled states obtained by ( $\mathbf{P}_{\text {OS-POD }}^{\ell}$ ) ought to differ. This is in fact the case as can be seen by comparing the POD basis elements associated to the uncontrolled state to those of the optimally controlled state in Figures 2 and Figure 3 for the choice $\ell=4$. In Figure 4 we present the POD basis which result from the optimal state when (4.1) is solved by applying the FE-SQP approach. These basis functions are very similar to those obtained by OS-POD. For POD not only the shape of the eigenfunctions but also the associated eigenvalues are of importance. In view of the above discussion and the interpretation of the POD-basis construction as reconstruction the energy of the system, see e.g. [5], it can be expected that the eigenvalues for the POD basis computed from the uncontrolled states decay more rapidly than those for the optimally controlled states. This is confirmed by the results in Table 1 which gives the results for the first four eigenvalues - scaled by the sum over all eigenvalues - for $n=0$, which coincides with the uncontrolled state, and for $n=4$. Moreover, in the last column of Table 1 we present the first four scaled eigenvalues that we obtain from the snapshots using the optimal FE controls. 

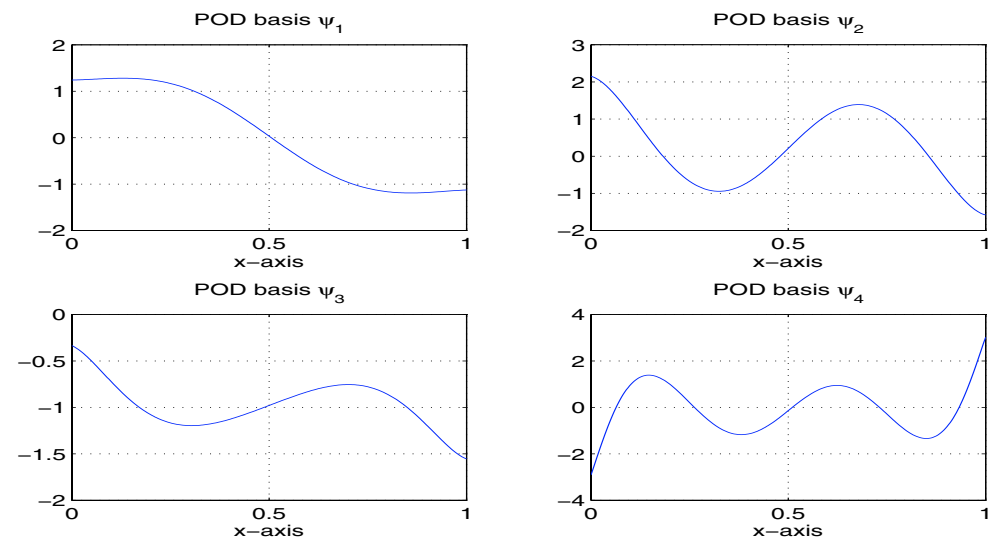

Figure 2. Run 1: POD basis functions associated with the uncontrolled Burgers equation, i.e., $u=v=0$.
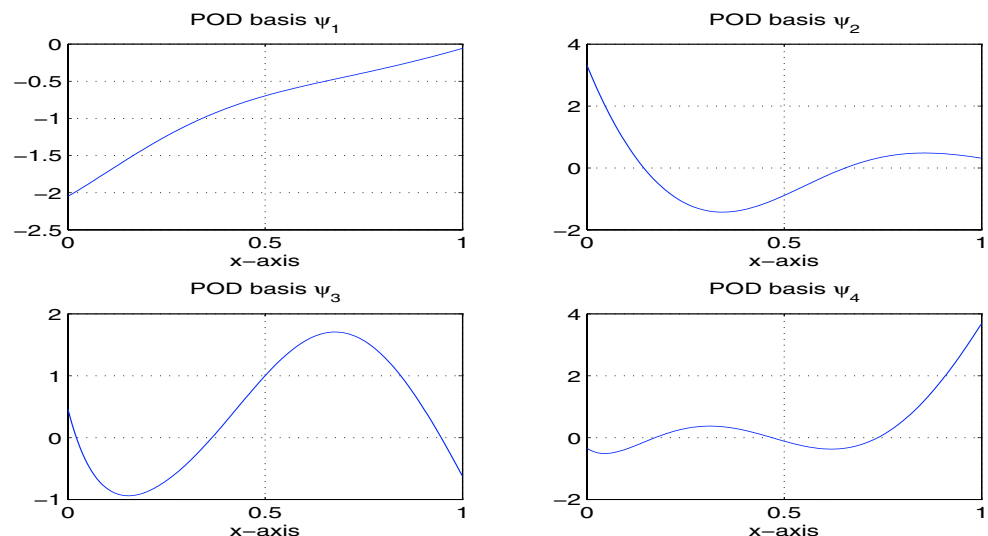

Figure 3. Run 1: POD basis functions associated with the final OS-POD update of the controls.
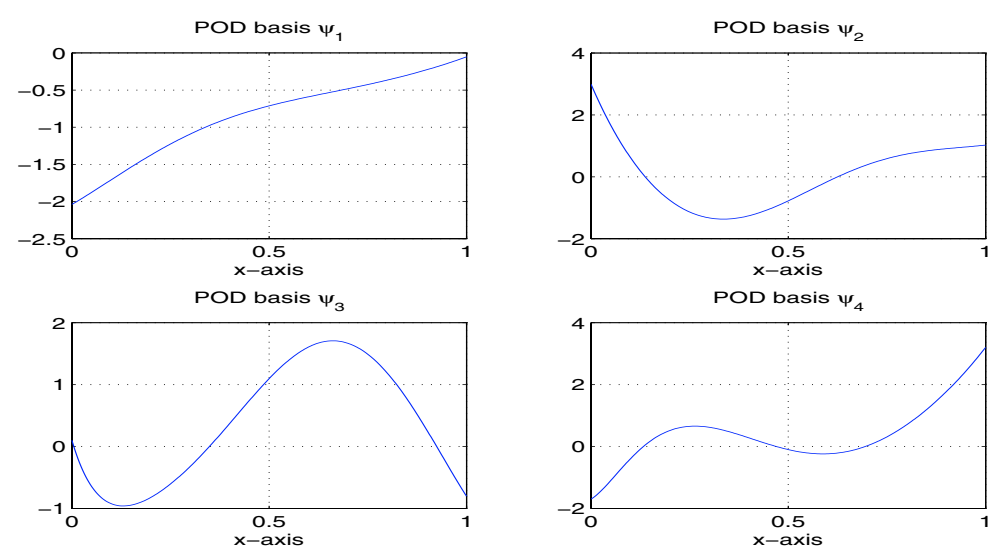

Figure 4. Run 1: POD basis functions associated with the optimal FE-SQP controls. 
TABLE 1. Run 1: decay of the first four eigenvalues $\lambda_{i}$ for the OS-POD strategy for $n=0$, $n=4$ and for the first four eigenvalues associated with the snapshots that are computed by using the optimal FE controls. For $n=0$ we have the decay of the first eigenvalues associated with the uncontrolled solution.

\begin{tabular}{|c||c|c|c|}
\hline & $n=0$ & $n=4$ & with FE controls \\
\hline$\lambda_{1} / \operatorname{tr}\left(\mathcal{K}_{h}\right)$ & 0.97187 & 0.87661 & 0.88092 \\
\hline$\lambda_{2} / \operatorname{tr}\left(\mathcal{K}_{h}\right)$ & 0.02209 & 0.08051 & 0.08734 \\
\hline$\lambda_{3} / \operatorname{tr}\left(\mathcal{K}_{h}\right)$ & 0.00579 & 0.02736 & 0.02744 \\
\hline$\lambda_{4} / \operatorname{tr}\left(\mathcal{K}_{h}\right)$ & 0.00025 & 0.00191 & 0.00292 \\
\hline
\end{tabular}

TABLE 2. Run 1: values of the cost functional for the different approaches (in brackets: value of the cost using POD solution in original system).

\begin{tabular}{|l||l|}
\hline & \multicolumn{1}{|c|}{$J(y, u, v)$} \\
\hline \hline Uncontrolled solution & 0.22134 \\
\hline OS-POD & $0.03813(0.03856)$ \\
\hline FE-SQP & 0.03765 \\
\hline
\end{tabular}
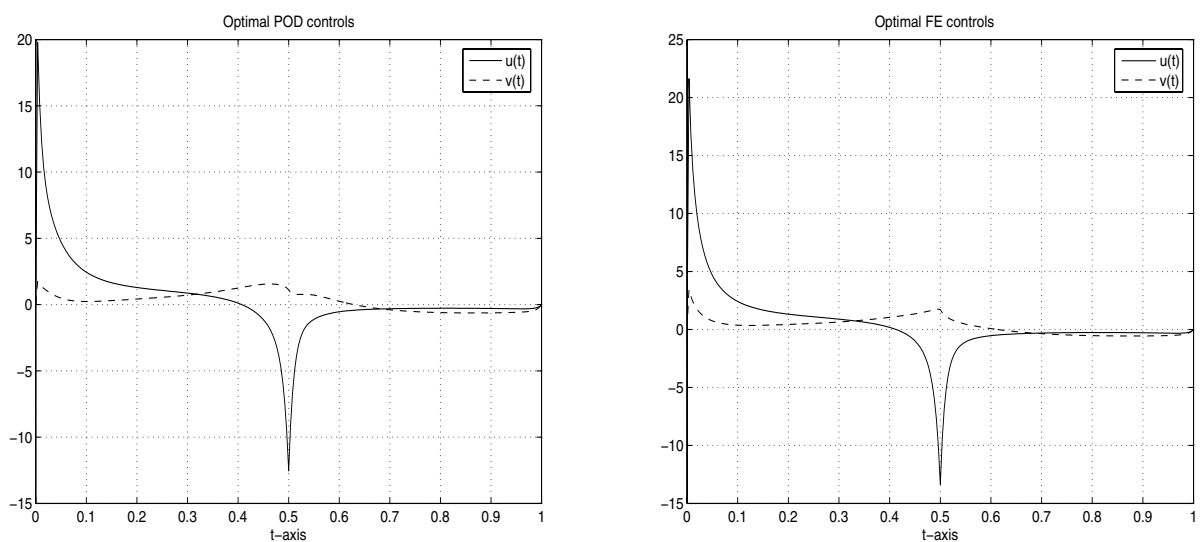

Figure 5. Run 1: optimal control for OS-POD strategy (left) and optimal FE-SQP controls (right).

In Table 2 the values of the cost functional are compared for different controls. First the value of the cost with control set to zero is given. Then the results for the optimal solution obtained by OS-POD and by FE-SQP are given. In parenthesis the value of the cost $J$ is depicted, where the state $y$ is computed from (4.1b)-(4.1e) with the optimal control from OS-POD. Note that the value for the cost obtained by OS-POD is close to that obtained from the FE-SQP solution. It is remarkable that for the optimal solution obtained by basic POD the values for the cost are $0.02254(0.27465)$ with the meaning of the number in parenthesis defined as for OS-POD in Table 2. The significant difference between these two values for the cost can be explained by the fact that the POD model on the basis of the uncontrolled solution is not a good approximation for (4.1b)-(4.1e).

Not only the values of the cost $J$ but also the optimal controls obtained from OS-POD and FE-SQP are very close as can be seen from Figure 5. As expected from the graph of $z$ the most critical point is $t=0.5$ for the control $u$. - Figure 6 shows the optimal state for OS-POD. The optimal state for FE-SQP is quite similar.

Compared to the basic POD approach, OS-POD requires an increased computational effort. It is therefore instructive to look at the distribution of the computing times for the steps involved in realizing the algorithm described at the beginning of this section. In Table 3 we provide the numbers for one typical iteration. 


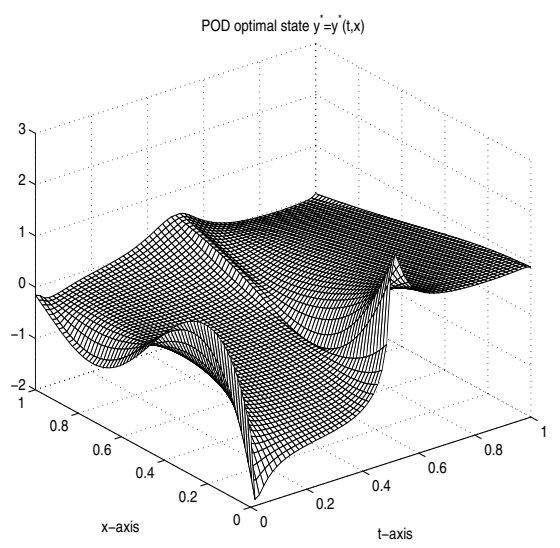

Figure 6. Run 1: optimal POD state for the OS-POD strategy.

TABLE 3. Run 1: averaged CPU times for one iteration of OS-POD.

\begin{tabular}{|l||c|}
\hline Partial steps & CPU time \\
\hline \hline Generate snapshots & $0.62 \mathrm{~s}$ \\
\hline POD computation & $0.09 \mathrm{~s}$ \\
\hline Compute ROM & $0.03 \mathrm{~s}$ \\
\hline SQP solve $\left(\mathbf{P}^{\ell}\right)$ & $14.16 \mathrm{~s}$ \\
\hline Compute $\mu_{i}$ 's & $2.07 \mathrm{~s}$ \\
\hline FE dual solve & $0.71 \mathrm{~s}$ \\
\hline Backtracking in step $(5)$ & $0.32 \mathrm{~s}$ \\
\hline
\end{tabular}

It takes on the order of $18 \mathrm{~s}$. OS-POD terminated after 4 iterations. These numbers should be compared to the FE-SQP solution for where the stopping criterion was met after seven SQP iterations and 445 seconds. Also for OS-POD, most of the computational effort results from the SQP-step for the reduced system.

While computing time is certainly a significant issue, we should mention that the reduced model associated to the optimal control may not be the final goal but rather only an intermediate step of a control theoretic investigation. This can be the case, for instance, in closed loop control, when the closed loop strategy is based on a reduced model for the open loop system. In this case the open loop model reduction step would be carried out off-line, whereas the closed loop control for the reduced order model would have to be solved in 'real-time'.

Run 2. The first example already shows that there can be a significant difference in the POD basis functions between the initial choice and the optimized basis. In the second example, again the uncontrolled solution and the desired state (see Fig. 7) are essentially different. For the uncontrolled state most of the energy is represented by just one mode; compare Table 4 . The desired state could also be presented by one mode, namely a constant function. Due to the initial condition and the discontinuity in time, the desired state is not exactly controllable by any choice of controls. Rather the dynamics of the optimally controlled state is rich in dynamical features, as can be seen from Figure 8. Still, $\ell=4$ basis functions capture $99.9 \%$ of the energy; compare Table 4 .

Let us now turn to the specifications in detail. We consider (4.1) with $T=1, \beta=0.001, \nu=0.75, \sigma_{0}=\nu / 2$, $\sigma_{1}=\nu, f(t, x)=x-2, y_{0}(x)=-x, z(t, x)=-\sin (2 \pi(t-T / 4)) / 2$ for $(t, x) \in[T / 4,3 T / 4] \times[0,1]$ and $z(t, x)=0$ otherwise, see Figure 7 (right). Note that $\phi(x)=x-2$ is a stationary solution to (4.1b) $-(4.1 \mathrm{~d})$ for $u=v=0$ and we have $y \rightarrow \phi$ as $t$ tends to $\infty$; compare Figure 7 (left). The POD basis associated with the uncontrolled state is plotted in Figure 9. Taking $\ell=4$ and applying OS-POD we obtain the POD basis depicted in Figure 10. 

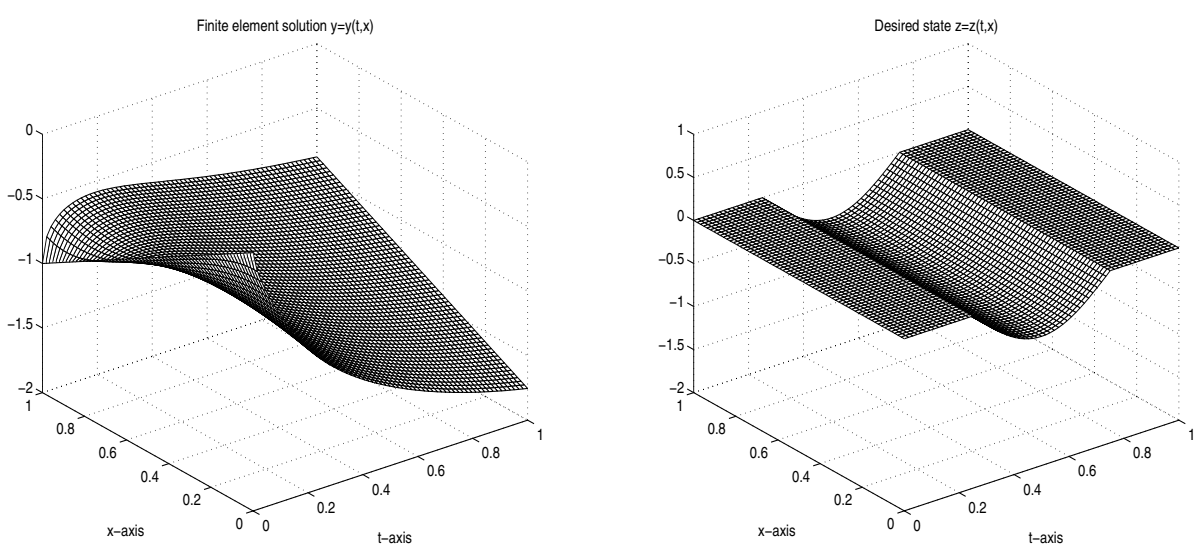

Figure 7. Run 2: FE solution to the uncontrolled Burgers equation, i.e., $u=v=0$ (left) and desired state (right).

TABLE 4. Run 2: decay of the first four eigenvalues $\lambda_{i}$ for the OS-POD strategy for $n=0$, $n=5$ and for the first four eigenvalues associated with the snapshots that are computed by using the optimal FE controls. For $n=0$ we have the decay of the first eigenvalues associated with the uncontrolled solution.

\begin{tabular}{|c||c|c|c|}
\hline & $n=0$ & $n=5$ & With FE controls \\
\hline$\lambda_{1} / \operatorname{tr}\left(\mathcal{K}_{h}\right)$ & 0.99261 & 0.84940 & 0.84464 \\
\hline$\lambda_{2} / \operatorname{tr}\left(\mathcal{K}_{h}\right)$ & 0.00737 & 0.13535 & 0.14117 \\
\hline$\lambda_{3} / \operatorname{tr}\left(\mathcal{K}_{h}\right)$ & 0.00002 & 0.01326 & 0.01236 \\
\hline$\lambda_{4} / \operatorname{tr}\left(\mathcal{K}_{h}\right)$ & 0.00000 & 0.00137 & 0.00114 \\
\hline
\end{tabular}

POD optimal state $y^{\prime}=y^{\prime}(t, x)$

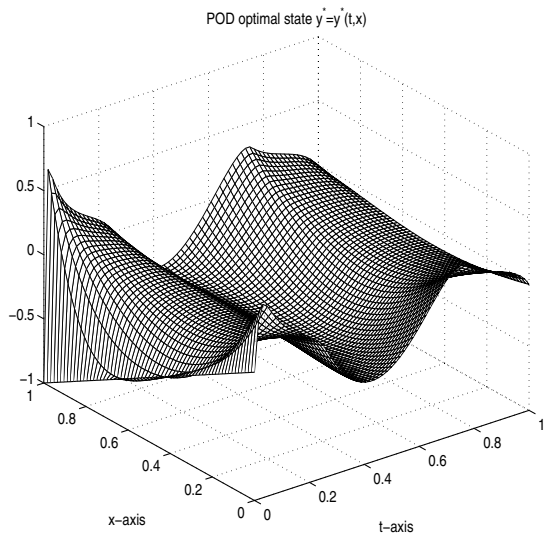

Figure 8. Run 2: OS-POD optimal state.

In Figure 11 we present the POD basis which result from the optimal state when (4.1) is solved by applying the FE-SQP approach. The first three basis functions are very similar to those obtained by OS-POD, whereas there are still some difference for the fourth POD basis near $x=0$. The OS-POD optimal state is shown in Figure 8. Table 4 gives the results for the first four eigenvalues. We point at that the difference in the decay rates between the uncontrolled and the optimally controlled POD eigenvalues. 

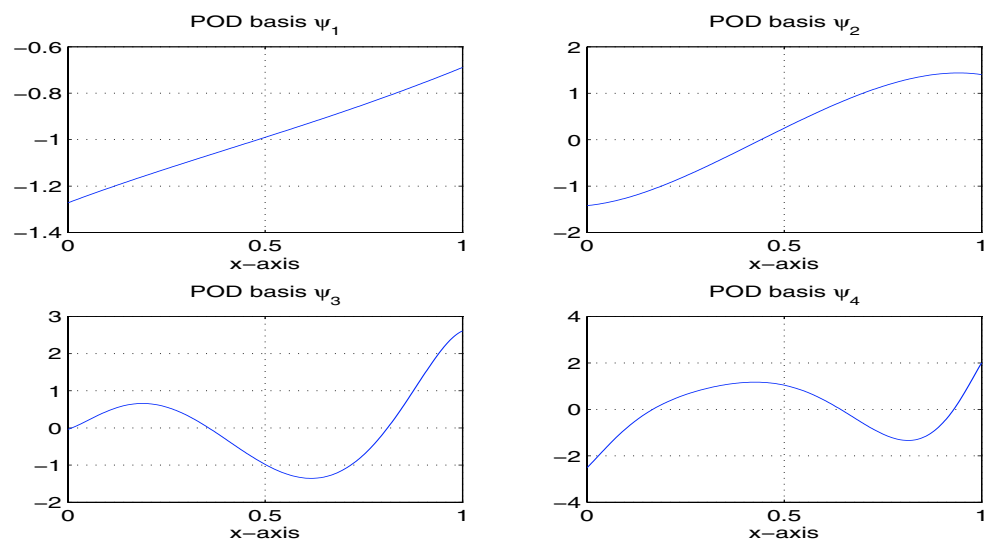

FiguRE 9. Run 2: POD basis functions associated with the uncontrolled Burgers equation, i.e., $u=v=0$.
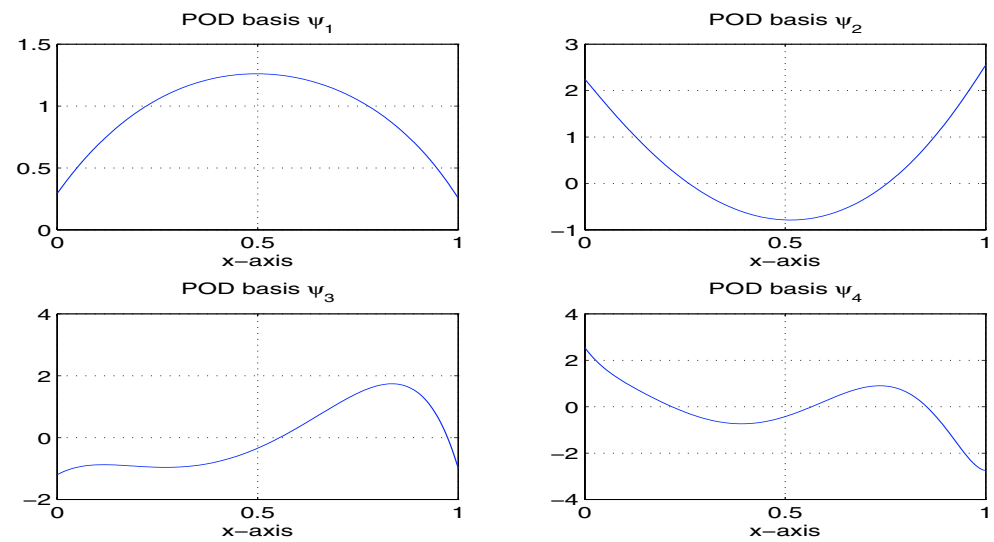

FIGURE 10. Run 2: POD basis functions associated with the final OS-POD update of the controls.
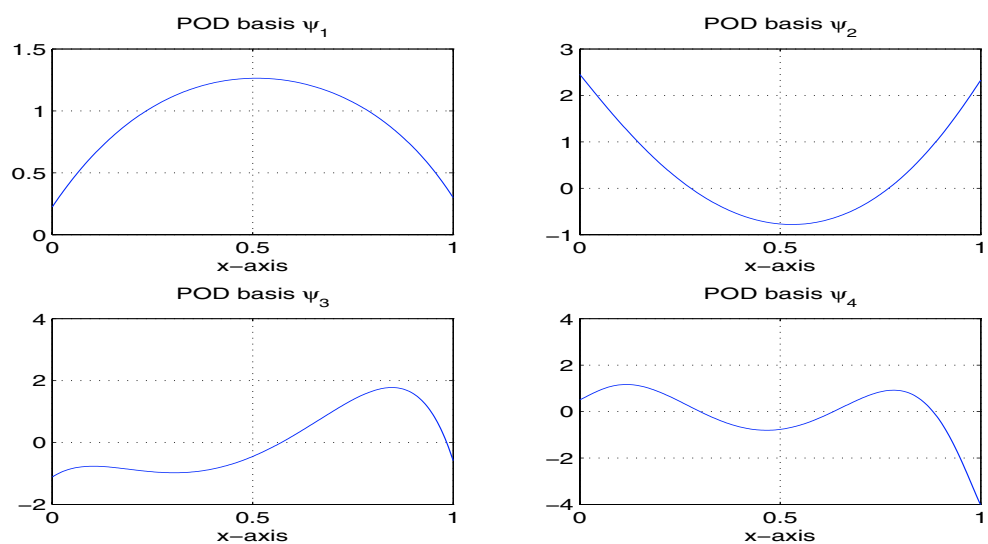

Figure 11. Run 2: POD basis functions associated with the optimal FE-SQP controls. 
TABLE 5. Run 2: values of the cost functional for the different approaches.

\begin{tabular}{|l||c|}
\hline & $J(y, u, v)$ \\
\hline \hline Uncontrolled solution & 0.41053 \\
\hline OS-POD & 0.01369 \\
\hline FE-SQP & 0.01368 \\
\hline
\end{tabular}

In Table 5 the values of the cost functional are compared for different controls. First the value of the cost with control set to zero is given. Then the results for the optimal solution obtained by OS-POD and by FE-SQP are given. Note that the value for the cost obtained by OS-POD is close to that obtained from the FE-SQP solution.

OS-POD terminated after 5 iterations. One OS-POD iteration requires in the order of $10 \mathrm{~s}$. The FE-SQP solution is computed after two SQP iterations in $101 \mathrm{~s}$.

\section{Appendix}

Proof of Theorem 2.2. Since $J^{\ell}$ is bounded from below, there exists a minimizing sequence $\left\{u^{n}\right\}_{n=1}^{\infty}$ which is bounded in $L^{2}\left(\mathbb{R}^{m}\right)$. By $(\mathbf{H 1})$ the sequence $\left\{y^{n}\right\}_{n=1}^{\infty}$ is bounded in $L^{2}(D(\mathcal{A})) \cap H^{1}(V)$, where $y^{n}=y\left(u^{n}\right)$. Clearly $\left\{\psi_{i}^{n}\right\}_{n=1}^{\infty}$ is bounded for each $i=1, \ldots, \ell$ and $\left\{\left\|\mathcal{R}\left(y^{n}\right)\right\|_{L(X)}\right\}_{n=1}^{\infty}$ is bounded, since $\left\{y^{n}\right\}_{n=1}^{\infty}$ is bounded in $L^{2}(V)$. This implies that $\left\{\lambda_{i}^{n}\right\}_{n=1}^{\infty}$ is bounded for every $i=1, \ldots, \ell$ as well. Hence there exist

$$
\left(y^{*}, u^{*}, \psi^{*}, \lambda^{*}\right) \in\left(L^{2}(D(\mathcal{A})) \cap H^{1}(V)\right) \times L^{2}\left(\mathbb{R}^{m}\right) \times X^{\ell} \times \mathbb{R}^{\ell}
$$

such that for a subsequence denoted by the same symbols

$$
\left(y^{n}, u^{n}, \psi_{i}^{n}, \lambda_{i}^{n}\right) \rightarrow\left(y^{*}, u^{*}, \psi_{i}^{*}, \lambda_{i}^{*}\right)
$$

weakly in $\left(L^{2}(D(\mathcal{A})) \cap H^{1}(V)\right) \times L^{2}\left(\mathbb{R}^{m}\right) \times X^{\ell} \times \mathbb{R}^{\ell}$. It follows that $y^{*}=y\left(u^{*}\right)$ is the solution to (2.1) with $u=u^{*}$. We next argue that $\lambda_{i}^{*}$ is an eigenvalue to $\mathcal{R}\left(y^{*}\right)$ with eigenvector $\psi_{i}^{*}$. By Aubin's lemma, $c f$. [6] and (H3) it follows that $y^{n} \rightarrow y^{*}$ strongly in $L^{2}(V)$. Hence for any $v \in X$

$$
\begin{gathered}
\lambda_{i}^{n}\left\langle\psi_{i}^{n}, v\right\rangle_{X}=\left\langle\mathcal{R}\left(y^{n}\right) \psi_{i}^{n}, v\right\rangle_{X}=\int_{0}^{T}\left\langle y^{n}(t), \psi_{i}^{n}\right\rangle_{X}\left\langle y^{n}(t), v\right\rangle_{X} \\
\rightarrow \int_{0}^{T}\left\langle y^{*}(t), \psi\right\rangle_{X}\left\langle y^{*}(t), v\right\rangle_{X}=\left\langle\mathcal{R}\left(y^{*}\right) \psi_{i}^{*}, v\right\rangle_{X} .
\end{gathered}
$$

Passing to the limit on the left implies that

$$
\lambda_{i}^{*} \psi_{i}^{*}=\mathcal{R}\left(y^{*}\right) \psi_{i}^{*} \quad \text { for } i=1, \ldots, \ell
$$

so that $\left(\psi_{i}^{*}, \lambda_{i}^{*}\right)$ are eigenvector-eigenvalue pairs for each $i=1, \ldots, \ell$.

We next argue that

$$
\psi_{i}^{n} \rightarrow \psi_{i}^{*} \text { strongly in } X \text { for each } i=1, \ldots, \ell \text {. }
$$

Passing to the limit in

implies that

$$
\lambda_{i}^{n}=\left\langle\mathcal{R}\left(y^{n}\right) \psi_{i}^{n}, \psi_{i}^{n}\right\rangle_{X}=\int_{0}^{T}\left\langle y^{n}(t), \psi_{i}^{n}\right\rangle_{X}^{2} \mathrm{~d} t
$$

$$
\lambda_{i}^{*}=\left\langle\mathcal{R}\left(y^{*}\right) \psi_{i}^{*}, \psi_{i}^{*}\right\rangle_{X}=\lambda_{i}^{*}\left\|\psi_{i}^{*}\right\|_{X}^{2}
$$


By (H4) we have $\lambda_{i}^{*}>0$ and hence $\left\|\psi_{i}^{*}\right\|_{X}=1$. Since

$$
1=\left\|\psi_{i}^{*}\right\|_{X} \leq \liminf _{n \rightarrow \infty}\left\|\psi_{i}^{n}\right\|_{X}=1
$$

it follows that $\lim _{n \rightarrow \infty}\left\|\psi_{i}^{n}\right\|_{X}=\left\|\psi_{i}^{*}\right\|_{X}$ and hence $\psi_{i}^{n} \rightarrow \psi_{i}^{*}$ strongly in $X$, for each $i=1, \ldots, \ell$. This also implies that

$$
\left\langle\psi_{i}^{*}, \psi_{j}^{*}\right\rangle_{X}=\delta_{i j} \quad \text { for all } i, j=1, \ldots, \ell .
$$

Before passing to the limit in

$$
E\left(\psi^{n}\right) \dot{x}^{n}(t)+A\left(\psi^{n}\right) x^{n}(t)+\mathfrak{N}\left(x^{n}(t), \psi^{n}\right)=B\left(\psi^{n}\right) u^{n}(t),
$$

we need to argue that

$$
\psi_{i}^{n} \rightarrow \psi_{i}^{*} \text { strongly in } V \text { for } i=1, \ldots, \ell,
$$

also in case $X=H$. Note that for the following argument we need $y^{n} \rightarrow y^{*}$ strongly in $L^{2}(V)$, which was not essential for the case $X=V$ so far. Let

$$
b_{n}=\frac{1}{\lambda_{i}^{n}}\left\langle y^{n}(\cdot), \psi_{i}^{n}\right\rangle_{H} \quad \text { and } \quad b^{*}=\frac{1}{\lambda_{i}^{*}}\left\langle y^{*}(\cdot), \psi_{i}^{*}\right\rangle_{H} \quad \text { in } L^{2}(\mathbb{R}),
$$

and note that $b_{n} \rightarrow b^{*}$ in $L^{2}(\mathbb{R})$. Therefore we have

$$
\left\|\psi_{i}^{n}-\psi_{i}^{*}\right\|_{V}=\int_{0}^{T}\left\|\left(b_{n}(t)-b^{*}(t)\right) y^{n}(t)+b^{*}(t)\left(y^{n}(t)-y^{*}(t)\right)\right\|_{V} \mathrm{~d} t \stackrel{n \rightarrow \infty}{\rightarrow} 0
$$

for $i=1, \ldots, \ell$.

Let us now turn to (A.2) with initial condition $x(0)=x_{0}$. We need to argue existence before we can pass to the limit. From (A.2) we have for $y^{\ell, n}=\sum_{i=1}^{\ell} x_{i}^{n} \psi_{i}^{n}$ and (H2)

$$
\begin{aligned}
\frac{1}{2} \frac{\mathrm{d}}{\mathrm{d} t}\left\|y^{\ell, n}(t)\right\|_{H}^{2}+\alpha\left\|y^{\ell, n}(t)\right\|_{V}^{2} \leq & \frac{\alpha}{2}\left\|y^{\ell, n}(t)\right\|_{V}^{2}+c_{2}\left\|y^{\ell, n}(t)\right\|_{H}^{2} \\
& +c_{3}+\|\mathcal{B}\|_{\mathcal{L}\left(\mathbb{R}^{m}, H\right)}\|u(t)\|_{\mathbb{R}^{m}}\left\|y^{\ell, n}(t)\right\|_{H},
\end{aligned}
$$

which by Gronwall's lemma implies the a priori estimate

$$
\left\|y^{\ell, n}\right\|_{C(H)} \leq K_{3}
$$

for a constant $K_{3}$ independent of $n$. Existence of a solution to (A.2) now follows by a local existence and extension argument.

Since $\lim _{n \rightarrow \infty} \psi_{i}^{n}=\psi_{i}^{*}$ in $V$ for $i=1, \ldots, \ell$ it follows that the $V, H$ and $V^{*}$ norms on $V^{\ell}\left(y^{n}\right)$ are uniformly equivalent. Hence $\left\{\left\|y^{\ell, n}\right\|_{C(V)}\right\}_{n=1}^{\infty}$ is uniformly bounded as well. Note that (A.2) can equivalently be expressed as

$$
\frac{\mathrm{d}}{\mathrm{d} t} y^{\ell, n}(t)+\mathcal{A} y^{\ell, n}(t)+P_{\mathrm{OSP}}^{\ell}\left(y^{n}\right) \mathcal{N}\left(y^{\ell, n}(t)\right)=\mathcal{B} u^{n}(t),
$$

for all $n$, where $P_{\mathrm{OSP}}^{\ell}\left(y^{n}\right)$ denotes the projection of $V^{*}$ onto $V^{\ell}\left(y^{n}\right) \subset V^{*}$. Since $\mathcal{N}$ maps bounded sets in $V$ onto bounded sets in $V^{*}$ by $(\mathbf{H 2})$, it follows that $\left\{\frac{\mathrm{d}}{\mathrm{d} t} y^{\ell, n}\right\}_{n=1}^{\infty}$ is bounded in $L^{2}\left(V^{*}\right)$ and hence also in $L^{2}(V)$, due to the uniform equivalence of the $V$ and $V^{*}$ norm on $V^{\ell}\left(y^{n}\right)$. This implies that $\left\{y^{\ell, n}\right\}_{n=1}^{\infty}$ is bounded in $H^{1}(V)$ and $\left\{x^{n}\right\}_{n=1}^{\infty}$ is bounded in $W^{1,2}\left(\mathbb{R}^{\ell}\right)$. Thus there exists a subsequence, denoted by the same symbol, 
and $x^{*} \in H^{1}\left(\mathbb{R}^{\ell}\right)$ such that $x^{n} \rightarrow x^{*}$ weakly in $H^{1}\left(\mathbb{R}^{\ell}\right)$. Together with continuity of $\mathcal{N}$ from $V$ to $V^{*}$ and strong convergence of $\psi_{i}^{n}$ to $\psi_{i}^{*}$ we can pass to the limit in (A.2) to obtain

$$
\left\{\begin{array}{l}
E\left(\psi^{*}\right) \dot{x}^{*}(t)+A\left(\psi^{*}\right) x^{*}(t)+\mathfrak{N}\left(x^{*}(t), \psi^{*}\right)=B\left(\psi^{*}\right) u^{*}(t) \\
x^{*}(0)=x_{0}
\end{array}\right.
$$

and thus $\left(y^{*}, x^{*}, \psi^{*}, \lambda^{*}, u^{*}\right)$ satisfy all constraints in ( $\mathrm{P}_{\text {OS-POD }}^{\ell}$. Passing to the limit in $J^{\ell}\left(x^{n}, \psi^{n}, u^{n}\right)$ we find

$$
J^{\ell}\left(x^{*}, \psi^{*}, u^{*}\right) \leq \lim _{n \rightarrow \infty} J^{\ell}\left(x^{n}, \psi^{n}, u^{n}\right),
$$

where $\left(x^{n}, \psi^{n}, u^{n}\right)$ is a minimizing sequence. Hence $\left(x^{*}, y^{*}, \psi^{*}, \lambda^{*}, u^{*}\right)$ is a solution to ( $\left.\mathrm{P}_{\mathrm{OS}-\mathrm{POD}}\right)$.

Proof of Theorem 2.3. We argue here that the linearization $e^{\prime}(\mathrm{z}): Z \rightarrow \Xi^{*}$ of $e$ at the point $\mathrm{z} \in Z$ is surjective operator. Then from duality theory, see e.g. [17], it follows that there exists a Lagrange multiplier $\xi \in \Xi$ with $\mathcal{L}_{\mathbf{z}}(\mathrm{z}, \xi)=0$. We have shown before the statement of Theorem 2.3 that this implies the desired optimality system.

Let $\zeta=\left(\bar{q}, \bar{q}_{0}, \bar{p}, \bar{p}_{0}, \bar{\mu}, \bar{\eta}\right)$ denote an arbitrary element of $\Xi^{*}$. We shall argue the existence of $\delta \mathbf{z}=$ $(\delta x, \delta y, \delta \psi, \delta \lambda, \delta u) \in Z$ such that

$$
e^{\prime}(\mathbf{z}) \delta \mathbf{z}=\zeta
$$

By assumption there exists $(\delta y, \delta u) \in W(0, T) \times L^{2}\left(\mathbb{R}^{m}\right)$ such that

$$
\begin{aligned}
e_{3}^{\prime}(\mathrm{z}) \delta \mathbf{z} & =\frac{\mathrm{d}}{\mathrm{d} t} \delta y+\mathcal{A} \delta y+\mathcal{N}^{\prime}(y) \delta y-\mathcal{B} \delta u=\bar{p}, \\
e_{4}^{\prime}(\mathrm{z}) \delta \mathbf{z} & =\delta y(0)=\bar{p}_{0} .
\end{aligned}
$$

With $\delta y$ fixed we turn to surjectivity of $\left(e_{5}^{\prime}(\mathbf{z}), e_{6}^{\prime}(\mathbf{z})\right)$ and consider for $i=1, \ldots, \ell$

$$
\begin{aligned}
\left(\mathcal{R}-\lambda_{i} I\right) \delta \psi_{i}+\delta \lambda_{i} \psi_{i} & =\bar{\mu}_{i}-\int_{0}^{T}\left\langle\delta y_{i}, \psi_{i}\right\rangle_{X} y_{i}-\int_{0}^{T}\left\langle y_{i}, \psi_{i}\right\rangle_{X} \delta y_{i} \\
2\left\langle\psi_{i}, \delta \psi_{i}\right\rangle_{X} & =\bar{\eta}_{i}
\end{aligned}
$$

Since $\operatorname{ker}\left(\mathcal{R}-\lambda_{i} I\right)=\operatorname{span}\left\{\psi_{i}\right\}$ this system has a unique solution $\left(\delta \psi_{i}, \delta \lambda_{i}\right) \in X \times \mathbb{R}$ for every $i=1, \ldots, m$. With $\delta \psi_{i}$ fixed we turn to the first two coordinates of $e^{\prime}(\mathbf{z}) \delta \mathbf{z}=\xi$ which are equivalent to the matrix ordinary differential equation

$$
\begin{aligned}
& E(\psi) \dot{\delta} x+A(\psi) \delta x+\mathfrak{N}_{x}(x, \psi) \delta x=\bar{q}-\left(e_{1}\right)_{\psi}(\mathbf{z}) \delta \psi \\
& E(\psi) \delta x(0)=\bar{q}_{0}-\left(e_{2}\right)_{\psi}(\mathbf{z}) \delta \psi
\end{aligned}
$$

which has a unique solution $\delta x \in H^{1}\left(\mathbb{R}^{\ell}\right)$. Thus $e^{\prime}(\mathrm{z})$ is surjective and the assertion follows.

Proof of Theorem 2.4. Utilizing Proposition 2.1 we argue that the relationship between normalized eigenvector $\psi_{i}$ of $\mathcal{R}=\mathcal{Y Y}^{*}$ and $\varphi_{i}$ of $\mathcal{K}=\mathcal{Y}^{*} \mathcal{Y}$ is given by

$$
\sqrt{\lambda_{i}} \psi_{i}=\mathcal{Y}^{*} \psi_{i} \quad \text { and } \quad \psi_{i}=\frac{1}{\sqrt{\lambda_{i}}} \mathcal{Y} \varphi_{i}
$$


To derive the optimality system for the "K $\mathcal{K}$-formulation" we proceed as in the " $\mathcal{R}$-formulation" and introduce the Lagrangian $\mathcal{L}^{\mathcal{K}}: Z \times \Xi \rightarrow \mathbb{R}$ by

$$
\begin{aligned}
\mathcal{L}^{\mathcal{K}}\left(\mathrm{z}, \xi^{\mathcal{K}}\right)= & J^{\ell}(x, \psi, u)+\left\langle e_{1}(\mathrm{z}), q^{\mathcal{K}}\right\rangle_{L^{2}\left(\mathbb{R}^{\ell}\right)}+\left\langle e_{2}(\mathbf{z}), q_{0}^{\mathcal{K}}\right\rangle_{\mathbb{R}^{\ell}} \\
& +\left\langle e_{3}(\mathbf{z}), p^{\mathcal{K}}\right\rangle_{L^{2}\left(V^{*}\right), L^{2}(V)}+\left\langle e_{4}(\mathrm{z}), p_{0}^{\mathcal{K}}\right\rangle_{H} \\
& +\sum_{i=1}^{\ell}\left\langle\left(\mathcal{K}-\lambda_{i} I\right) \varphi_{i}, \mu_{i}^{\mathcal{K}}\right\rangle_{L^{2}(\mathbb{R})}+\sum_{i=1}^{\ell}\left(\left\|\varphi_{i}\right\|_{L^{2}(\mathbb{R})}^{2}-1\right) \eta_{i}^{\mathcal{K}},
\end{aligned}
$$

where $\varphi_{i}=\frac{1}{\sqrt{\lambda_{i}}} \mathcal{Y}^{*} \psi_{i}$. In the context of the general setting this means that $e_{5}$ and $e_{6}$ are replaced by

$$
\begin{aligned}
& \left(e_{5}^{\mathcal{K}}(\mathrm{z})\right)_{i}=\frac{1}{\sqrt{\lambda_{i}}}\left(\mathcal{K}-\lambda_{i} I\right) \mathcal{Y}^{*} \psi_{i} \\
& \left(e_{6}^{\mathcal{K}}(\mathrm{z})\right)_{i}=\frac{1}{\lambda_{i}}\left\|\mathcal{Y}^{*} \psi_{i}\right\|^{2}-1
\end{aligned}
$$

Existence of a Lagrange multiplier $\xi^{\mathcal{K}}$ follows from surjectivity of the linearisation of the equivalently constraints which is argued as in the proof of Theorem 2.3. Hence the optimality system is obtained from $\mathcal{L}_{\mathbf{z}}^{\mathcal{K}}\left(\mathbf{z}, \xi^{\mathcal{K}}\right)=0$. From $\mathcal{L}_{\mathbf{z}}^{\mathcal{K}}\left(\mathbf{z}, \xi^{\mathcal{K}}\right)=0$ we obtain $(2.16)$, i.e., the equations for $q$ and $q^{\mathcal{K}}$ coincide. Utilizing $\mathcal{L}_{y}^{\mathcal{K}}\left(\mathbf{z}, \xi^{\mathcal{K}}\right)=0$ we find (2.20). In fact let us just consider one of the terms $\left\langle\left(\mathcal{K}-\lambda_{i} I\right) \varphi_{i}, \mu_{i}^{\mathcal{K}}\right\rangle_{L^{2}(\mathbb{R})}$. We find

$$
\begin{aligned}
\frac{\partial}{\partial y}\left\langle\left(\mathcal{K}-\lambda_{i} I\right) \varphi_{i}, \mu_{i}^{\mathcal{K}}\right\rangle_{L^{2}(\mathbb{R})}= & \mu_{i}^{\mathcal{K}}(t) \int_{0}^{T} \mathcal{I}^{-1} y(s, \cdot) \varphi_{i}(s) \mathrm{d} s \\
& +\varphi_{i}(t) \int_{0}^{T} \mathcal{I}^{-1} y(s, \cdot) \mu_{i}^{\mathcal{K}}(s) \mathrm{d} s
\end{aligned}
$$

which enters into the right hand side of (2.20). From $\mathcal{L}_{u}^{\mathcal{K}}\left(\mathrm{z}, \xi^{\mathcal{K}}\right)=0$ we find $(2.22)$. Finally $\mathcal{L}_{\psi_{i}, \lambda_{i}}^{\mathcal{K}}\left(\mathrm{z}, \xi^{\mathcal{K}}\right)$ for $i=1, \ldots, \ell$ imply

$$
\begin{aligned}
\mathcal{Y}\left(\mathcal{K}-\lambda_{i} I\right) \mu_{i}^{\mathcal{K}}+2 \eta_{i}^{\mathcal{K}} \mathcal{Y} \varphi_{i}+\sqrt{\lambda_{i}} \mathcal{I} \mathcal{G}_{i} & =0 \\
\left\langle\varphi_{i}, \mu_{i}^{\mathcal{K}}\right\rangle_{L^{2}(\mathbb{R})} & =0 .
\end{aligned}
$$

Taking the inner product of (A.4) with $\psi_{i}$ and using (A.5) we obtain

$$
\eta_{i}^{\mathcal{K}}=-\frac{1}{2}\left\langle\mathcal{G}_{i}, \psi_{i}\right\rangle_{X^{*}, X}
$$

so that $\eta_{i}^{\mathcal{K}}$ and $\eta_{i}$ coincide. From (A.4) we have

$$
\mathcal{K}\left(\mathcal{K}-\lambda_{i} I\right) \mu_{i}^{\mathcal{K}}=-\tilde{\mathcal{G}}_{i}-2 \eta_{i} \mathcal{K} \varphi_{i}
$$

where $\tilde{G}_{i}=\sqrt{\lambda_{i}} \mathcal{Y}^{*} \mathcal{I} \mathcal{G}_{i}$. Since $\mathcal{K}^{-1}\left(\tilde{\mathcal{G}}_{i}+2 \eta_{i} \mathcal{K} \varphi_{i}\right)$ is orthogonal to $\operatorname{ker}\left(\mathcal{K}-\lambda_{i} I\right)=\operatorname{span}\left\{\varphi_{i}\right\}$ we have the desired result. 


\section{REFERENCES}

[1] K. Afanasiev and M. Hinze, Adaptive control of a wake flow using proper orthogonal decomposition, in Lecture Notes in Pure and Applied Mathematics 216, Marcel Dekker (2001) 317-332.

[2] E. Arian, M. Fahl and E. Sachs, Trust-region proper orthogonal decomposition for flow control. Technical Report 2000-25, ICASE (2000).

[3] P. Astrid, S. Weiland, K. Willcox and T. Backx, Missing point estimation in models described by proper orthogonal decomposition, in 43rd IEEE Conference on Decision and Control, Paradise Island, Bahamas (2004).

[4] H.T. Banks, M.L. Joyner, B. Winchesky and W.P. Winfree, Nondestructive evaluation using a reduced-order computational methodology. Inverse Problems 16 (2000) 1-17.

[5] G. Berkooz, P. Holmes and J.L. Lumley, Turbulence, Coherent Structures, Dynamical Systems and Symmetry. Cambridge Monographs on Mechanics, Cambridge University Press (1996).

[6] P. Constantin and C.Foias, Navier-Stokes Equations. Chicago Lectures in Mathematics, University of Chicago Press, Chicago (1989).

[7] M.A. Grepl and A.T. Patera, A posteriori error bounds for reduced-basis approximations of parametrized parabolic partial differential equations. ESAIM: M2AN 39 (2005) 157-181.

[8] S. Gugercin and A.C. Antoulas, A survey of model reduction by balanced truncation and some new results. Int. J. Control 77 (2004) 748-766.

[9] T. Henri, Réduction de modéles par des méthodes de décomposition orthogonal propre. Ph.D. thesis, Université de Rennes, France (2004).

[10] C. Homescu, L.R. Petzold and R. Serban, Error estimation for reduced order models of dynamical systems. SIAM J. Numer. Anal. 43 (2005) 1693-1714.

[11] K. Ito and S.S. Ravindran, Reduced basis method for unsteady viscous flows. Int. J. Comp. Fluid Dynam. 15 (2001) 97-113.

[12] K. Kunisch and S. Volkwein, Control of Burgers' equation by a reduced order approach using proper orthogonal decomposition. J. Optim. Theor. Appl. 102 (1999) 345-371.

[13] K. Kunisch and S. Volkwein, Galerkin proper orthogonal decomposition methods for a general equation in fluid dynamics. SIAM J. Numer. Anal. 40 (2002) 92-515.

[14] K. Kunisch, S. Volkwein and L. Xie, HJB-POD based feedback design for the optimal control of evolution problems. SIAM J. Appl. Dynam. Syst. 4 (2004) 701-722.

[15] S. Lall, J.E. Marsden and S. Glavaški, A subspace approach to balanced truncation for model reduction of nonlinear control systems. Int. J. Robust Nonlinear Control 12 (2002) 519-535.

[16] H.V. Ly and H.T. Tran, Proper orthogonal decomposition for flow calculations and optimal control in a horizontal CVD reactor. Quarterly Appl. Math. 60 (2002) 631-656.

[17] H. Maurer and J. Zowe, First and second order necessary and sufficient optimality conditions for infinite-dimensional programming problems. Math. Programming 16 (1979) 98-110.

[18] B.C. Moore, Principal component analysis in linear systems: controllability, observability, and model reduction. IEEE Trans. Automatic Control AC-26 (1981) 17-31.

[19] S.S. Ravindran, Adaptive reduced-order controllers for a thermal flow system using proper orthogonal decomposition. SIAM J. Sci. Comput. 23 (2002) 1924-1942.

[20] D.V. Rovas, L. Machiels and Y. Maday, Reduced-basis output bound methods for parabolic problems. IMA J. Numer. Anal. 26 (2006) 423-445.

[21] C.W. Rowley, Model reduction for fluids using balanced proper orthogonal decomposition. Int. J. Bifurcation Chaos 15 (2005) 997-1013.

[22] L. Sirovich, Turbulence and the dynamics of coherent structures, parts I-III. Quarterly Appl. Math. XLV (1987) 561-590.

[23] K.Y. Tan, W.R. Graham and J. Peraire, Active flow control using a reduced order model and optimum control. AIAA (1996).

[24] R. Temam, Infinite-Dimensional Dynamical Systems in Mechanics and Physics. Springer Verlag, Berlin (1988).

[25] S. Volkwein, Second-order conditions for boundary control problems of the Burgers equation. Control Cybern. 30 (2001) 249-278.

[26] S. Volkwein, Boundary control of the Burgers equation: optimality conditions and reduced-order approach, in Optimal Control of Complex Structures, K.-H. Hoffmann, I. Lasiecka, G. Leugering, J. Sprekels and F. Tröltzsch Eds., International Series of Numerical Mathematics 139 (2001) 267-278.

[27] S. Volkwein, Lagrange-SQP techniques for the control constrained optimal boundary control problems for the Burgers equation. Comput. Optim. Appl. 26 (2003) 253-284.

[28] K. Willcox and J. Peraire, Balanced model reduction via the proper orthogonal decomposition, in 15th AIAA Computational Fluid Dynamics Conference, Anaheim, USA (June 2001). 